Y. Kitaoka

Nagoya Math. J.

Vol. 141 (1996), 1-27

\title{
THE MINIMUM AND THE PRIMITIVE REPRESENTATION OF POSITIVE DEFINITE QUADRATIC FORMS II
}

\author{
YOSHIYUKI KITAOKA
}

We are concerned with representation of positive definite quadratic forms by a positive definite quadratic form. Let us consider the following assertion

$\mathrm{A}_{m, n}$ : Let $M, N$ be positive definite quadratic lattices over $\mathbf{Z}$ with $\operatorname{rank}(M)=m$ and $\operatorname{rank}(N)=n$ respectively. We assume that the localization $M_{p}$ is represented by $N_{p}$ for every prime $p$, that is there is an isometry from $M_{p}$ to $N_{p}$. Then there exists a constant $c(N)$ dependent only on $N$ so that $M$ is represented by $N$ if $\min (M)>c(N)$, where $\min (M)$ denotes the least positive number represented by $M$.

We know that the assertion $A_{m, n}$ is true if $n \geq 2 m+3$. A suceeding natural problem is whether it is the best or not. It is known that this is the best if $m=1$, that is $A_{1,4}$ is false. But in the case of $m \geq 2$, what we know at present, is that there is an example $N$ so that $\mathrm{A}_{m, n}$ is false if $n-m=3$. We do not know such examples when $n-m=4$. Anyway, analyzing the counter-example, we come to the following two assertions $\mathrm{APW}_{m, n}$ and $\mathrm{R}_{m, n}$.

$\mathrm{APW}_{m, n}$ : There exists a constant $c^{\prime}(N)$ dependent only on $N$ so that $M$ is represented by $N$ if $\min (M)>c^{\prime}(N)$ and $M_{p}$ is primitively represented by $N_{p}$ for every prime $p$.

$\mathrm{R}_{m, n}$ : There is a lattice $M^{\prime}$ containing $M$ such that $M_{p}^{\prime}$ is primitively represented by $N_{p}$ for every prime $p$ and $\min \left(M^{\prime}\right)$ is still large if $\min (M)$ is large.

If the assertion $\mathrm{R}_{m, n}$ is true, then the assertion $\mathrm{A}_{m, n}$ is reduced to the apparently weaker assertion $\mathrm{APW}_{m, n}$. If the assertion $\mathrm{R}_{m, n}$ is false, then it becomes possible to make a counter-example to the assertion $A_{m, n}$. As a matter of fact, $\mathrm{APW}_{1,4}$ is true but $\mathrm{R}_{1,4}$ is false in general, and it yields examples of $N$ such that $\mathrm{A}_{1,4}$ is false.

We proved that the assertion $\mathrm{R}_{m, 2 m+1}$ (resp. $\mathrm{R}_{m, 2 m+2}$ ) is true if $m \geq 3$ (resp. $m$ $\geq 2$ ), respectively. The aim of this paper is study the case of $n=2 m$ for $m \geq 4$. In Section 1 , we study $\min \sum_{i=1}^{t}\left\lceil b r_{i} / N\right\rceil^{2} q_{i}$ where $q_{i}$ is a positive number, $r_{i}, N$ are integers, $b$ runs over integers $\not \equiv 0 \bmod N$ and $\lceil x\rceil(-0.5 \leq\lceil x\rceil<0.5)$

Received November 11, 1994. 
denotes the decimal part of $x$. In Section 2, we study the distribution of isotropic vectors on a quadratic space over a finite field. In Section 3 the transformation matrix of two specified basis $\left\{v_{1}, \ldots, v_{m}\right\},\left\{w_{1}, \ldots, w_{m}\right\}$ of a positive definite quadratic forms over $\mathbf{Z}$ is studied, where $\left(B\left(v_{i}, v_{j}\right)\right)$ is reduced in the sense of Minkowski and $\left(B\left(w_{i}, w_{j}\right)\right)$ gives a Jordan splitting at a prime $p$. In Section 4 , we show the assertion $\mathrm{R}_{m, 2 m}(m \geq 6)$ is true.

We denote by $\mathbf{Z}, \mathbf{Q}, \mathbf{Z}_{p}$ and $\mathbf{Q}_{p}$ the ring of integers, the field of rational numbers and their $p$-adic completions.

Terminology and notation on quadratic forms are those from [3]. We denote a quadratic form and the associated bilinear form by $Q$ and $B(B(x, x)=$ $Q(x))$ respectively. For a lattice on $M$ on a quadratic space $V$ over $\mathbf{Q}$, the scale $s(M)$ denotes $\{B(x, y) \mid x, y \in M\}$ and the norm $n(M)$ denotes a $\mathbf{Z}$-module spanned by $\{Q(x) \mid x \in M\}$. Even for the localization $M_{p}$ they are similarly defined. $\mathrm{d} M, \mathrm{~d} M_{p}$ denote the discriminant of $M, M_{p}$ respectively. A positive lattice means a lattice on a positive definite quadratic space over $\mathbf{Q}$. For a real number $x,[x]$ denotes the largest integer which does not exceed $x$.

\section{Minimum}

Definition. For a real number $x$, we define the decimal part $\lceil x\rceil$ by the conditions

$$
-1 / 2 \leq\lceil x\rceil<1 / 2 \text { and } x-\lceil x\rceil \in \mathbf{Z}
$$

Note that $\lceil x\rceil^{2}=\lceil-x\rceil^{2}$ for every real number $x$.

Definition. For positive numbers $a, b$, we write

$$
a \ll_{m} b
$$

if there is a positive number $c$ dependent only on $m$ such that $a / b<c$. If both $a$ $\ll_{m} b$ and $b \ll_{m} a$ hold, then we write

$$
a \asymp_{m} b .
$$

If $m$ is an absolute constant, then we omit $m$.

Definition. For positive numbers $c_{1}, c_{2}$, we say that a positive definite mat$\operatorname{rix} S^{(m)}=\left(s_{i, j}\right)$ is $\left(c_{1}, c_{2}\right)$-diagonal if we have

$$
c_{1} \operatorname{diag}\left(s_{1,1}, \cdots, s_{m, m}\right)<S<c_{2} \operatorname{diag}\left(s_{1,1}, \cdots, s_{m, m}\right) .
$$


If $S$ is reduced in the sense of Minkowski or in a Siegel domain $\widetilde{S}$, then $S$ is $\left(c_{1}\right.$, $c_{2}$ )-diagonal for some positive numbers $c_{1}, c_{2}$ (see Ch. 2 in [3]).

Lemma 1. Let $M=\mathbf{Z}\left[v_{1}, \ldots, v_{m}\right]$ be a positive lattice and assume that $\left(B\left(v_{i}\right.\right.$, $\left.v_{j}\right)$ ) is $\left(c_{1}, c_{2}\right)$-diagonal. For a primitive element $w=\sum_{i=1}^{m} r_{i} v_{i}$ in $M$ and for a natural number $N$, we have

$$
\min (M+\mathbf{Z}[w / N]) \asymp_{c_{1}, c_{2}} \min \left(\min (M), \min _{b \in \mathbf{Z}, N \nmid b} \sum_{i=1}^{m}\left\lceil b r_{i} / N\right\rceil^{2} Q\left(v_{i}\right)\right) .
$$

Proof. Since there are positive constants $c_{1}, c_{2}$ so that

$$
c_{1} \sum_{i=1}^{m} x_{i}^{2} Q\left(v_{i}\right)<Q\left(\sum_{i=1}^{m} x_{i} v_{i}\right)<c_{2} \sum_{i=1}^{m} x_{i}^{2} Q\left(v_{i}\right),
$$

putting

$$
Q^{\prime}\left(\sum_{i=1}^{m} x_{i} v_{i}\right):=\sum_{i=1}^{m} x_{i}^{2} Q\left(v_{i}\right)
$$

we have

$$
\begin{aligned}
\min _{Q}(M+\mathbf{Z}[w / N]) & \asymp_{c_{1}, c_{2}} \min _{Q^{\prime}}(M+\mathbf{Z}[w / N]) \\
& =\min \left(\sum_{i=1}^{m}\left(b_{\imath}+b r_{i} / N\right)^{2} Q\left(v_{\imath}\right)\right),
\end{aligned}
$$

where integers $b, b_{i}(i=1, \ldots, m)$ should satisfy $b_{i}+b r_{i} / N \neq 0$ for some $i$. By noting that under the restriction $N \mid b$, the minimum is equal to $\min _{Q^{\prime}}(M)$, and that the condition $N \nmid b$ yields $b_{i}+b r_{i} / N \neq 0$ for some $i$ because of the primitivity of $w$ in $M$, the above is equal to

$$
\min \left(\min (M), \min _{b \in \mathbf{Z}, N \nmid b} \sum_{i=1}^{m}\left\lceil b r_{i} / N\right\rceil^{2} Q\left(v_{i}\right)\right)
$$

Remark. Let $M$ and $M^{\prime}$ be positive lattices of $\operatorname{rank} M=\operatorname{rank} M^{\prime}$. Then the condition $M^{\prime} \supset M$ implies $\min \left(M^{\prime}\right) \leq \min (M) \leq\left[M^{\prime}: M\right]^{2} \min \left(M^{\prime}\right)$.

LEMMA 2. Suppose that $\min _{b \in \mathbf{Z}, N \times b} \sum_{i=1}^{m}\left\lceil b r_{i} / N\right\rceil^{2} Q\left(v_{i}\right)$ in Lemma 1 is attained at $b=B$ and then putting $N^{\prime}=(B, N)$, we have

$$
\begin{aligned}
\min (M+\mathbf{Z}[w / N]) & \asymp_{c_{1}, c_{2}} \min \left(M+\mathbf{Z}\left[w /\left(N / N^{\prime}\right)\right]\right) \\
& \varlimsup_{c_{1}, c_{2}} \min \left(\min (M), \min _{\substack{b \in \mathbf{Z} \\
\left(b, N / N^{\prime}\right)=1}} \sum_{i=1}^{m}\left\lceil b r_{\imath} /\left(N / N^{\prime}\right)\right\rceil^{2} Q\left(v_{\imath}\right)\right) .
\end{aligned}
$$


Proof. By virtue of

$$
\begin{aligned}
& \min _{b \in \mathbf{Z}, N \nmid b}\left(\sum_{i=1}^{m}\left\lceil b r_{i} / N\right\rceil^{2} Q\left(v_{i}\right)\right)=\min _{(b, N)=N^{\prime}}\left(\sum_{i=1}^{m}\left\lceil b r_{i} / N\right\rceil^{2} Q\left(v_{i}\right)\right) \\
& =\min _{\left(b, N / N^{\prime}\right)=1}\left(\sum_{i=1}^{m}\left\lceil b r_{i} /\left(N / N^{\prime}\right)\right\rceil^{2} Q\left(v_{i}\right)\right) \geq \min _{b \in \mathbf{Z},\left(N / N^{\prime}\right) \ngtr b}\left(\sum_{i=1}^{m}\left\lceil b r_{i} /\left(N / N^{\prime}\right)\right\rceil^{2} Q\left(v_{i}\right)\right),
\end{aligned}
$$

we have

$$
\begin{aligned}
& \min (M+\mathbf{Z}[w / N]) \asymp_{c_{1}, c_{2}} \min \left(\min (M), \min _{b \in \mathbf{Z}, N \nmid b} \sum_{i=1}^{m}\left\lceil b r_{i} / N\right\rceil^{2} Q\left(v_{i}\right)\right) \\
& =\min \left(\min (M), \min _{\left(b, N / N^{\prime}\right)=1} \sum_{i=1}^{m}\left\lceil b r_{\imath} /\left(N / N^{\prime}\right)\right\rceil^{2} Q\left(v_{i}\right)\right) \\
& \geq \min \left(\min (M), \min _{b \in \mathbf{Z},\left(N / N^{\prime}\right) \times b} \sum_{i=1}^{m}\left\lceil b r_{i} /\left(N / N^{\prime}\right)\right\rceil^{2} Q\left(v_{i}\right)\right) \\
& \gg c_{c_{1}, c_{2}} \min \left(M+\mathbf{Z}\left[w /\left(N / N^{\prime}\right)\right]\right) \\
& \geq \min (M+\mathbf{Z}[w / N]),
\end{aligned}
$$

because of $M+\mathbf{Z}[w / N] \supset M+\mathbf{Z}\left[w /\left(N / N^{\prime}\right)\right]$.

Lemma 3. Let $\alpha_{i}$ be positive numbers with $\alpha_{i}<1 / 2$ for $i=1, \ldots, t$ and $N a$ natural number. Put

$$
X\left(\alpha_{1}, \ldots, \alpha_{t} ; N\right):=\left\{\begin{array}{l|l}
\left(r_{1}, \ldots, r_{t}\right) \bmod N \mid \begin{array}{l}
\left|\left\lceil r r_{i} / N\right\rceil\right|<\alpha_{i} \text { for } i=1, \ldots, t \text { and } \\
\text { for some integer } r \text { with }(r, N)=1
\end{array}
\end{array}\right\} .
$$

Then we have

$$
\left|X\left(\alpha_{1}, \ldots, \alpha_{t} ; N\right)\right|<3^{t} N \prod_{i=1}^{t} \max \left(\alpha_{i} N, 1\right)
$$

Proof. Suppose that $\left(r_{1}, \ldots, r_{t}\right)$ is an element in $X\left(\alpha_{1}, \ldots, \alpha_{t} ; N\right)$ and $\left|\left\lceil r r_{i} / N\right\rceil\right|<\alpha_{i}$ for some integer $r$ relatively prime to $N$. We can choose integer $b_{i}$ so that $b_{i} \equiv r r_{i} \bmod N$ and $\left|b_{i} / N\right|<\alpha_{i}$. Then we have $r_{\imath} \equiv R b_{\imath} \bmod N$ for an integer $R$ with $r R \equiv 1 \bmod N$, and hence

$$
\begin{aligned}
|X| & \leq N\left|\left\{\left(b_{1} \ldots, b_{t}\right) \bmod N|| b_{i} / N \mid<\alpha_{i}(i=1, \ldots, t)\right\}\right| \\
& \leq N \prod_{i=1}^{t}\left(2\left[\alpha_{i} N\right]+1\right)<3^{t} N \prod_{i=1}^{t} \max \left(\alpha_{i} N, 1\right) .
\end{aligned}
$$

Proposition 1. Let $q_{1}, \ldots, q_{t}, c$ be positive numbers with $c / q_{i}<1 / 4$ for $i=$ $1, \ldots, t$, and $N$ and $N^{\prime}$ a natural number and a divisor of $N$, respectively. Let $S$ be a 
subset of $(\mathbf{Z} / N \mathbf{Z})^{t}$ such that for every element $\left(r_{1}, \ldots, r_{t}\right) \in S$

$$
\min _{b \in \mathbf{Z}, N \rtimes b}\left(\sum_{i=1}^{t}\left\lceil b r_{i} / N\right\rceil^{2} q_{i}\right)
$$

is given at $b$ with $N^{\prime}=(b, N)$. If

$$
\left|S \bmod N / N^{\prime}\right|>3^{t}\left(N / N^{\prime}\right) \Pi \max \left(\sqrt{c / q_{i}} \cdot N / N^{\prime}, 1\right),
$$

then there exists an element $\left(r_{1}, \ldots, r_{t}\right) \in S$ such that

$$
\min _{b \in \mathbf{Z}, N \times b}\left(\sum_{i=1}^{t}\left\lceil b r_{i} / N\right\rceil^{2} q_{i}\right) \geq c .
$$

Proof. Suppose that the assertion is false; then for every $\left(r_{1}, \ldots, r_{t}\right) \in S$

$$
\min _{b \in \mathbf{Z}, N \times b}\left(\sum_{i=1}^{t}\left\lceil b r_{i} / N\right\rceil^{2} q_{i}\right)<c
$$

where the minimum is given at $b$ with $N^{\prime}=(b, N)$. This yields

$$
\begin{aligned}
& \min _{b \in \mathbf{Z}, N \times b}\left(\sum_{i=1}^{t}\left\lceil b r_{i} / N\right\rceil^{2} q_{i}\right)=\min _{(b, N)=N^{\prime}}\left(\sum_{i=1}^{t}\left\lceil b r_{i} / N\right\rceil^{2} q_{i}\right) \\
= & \min _{\left(b, N / N^{\prime}\right)=1}\left(\sum_{i=1}^{t}\left\lceil b r_{i} /\left(N / N^{\prime}\right)\right\rceil^{2} q_{i}\right)<c
\end{aligned}
$$

and hence $\left(r_{1}, \ldots, r_{t}\right) \bmod \left(N / N^{\prime}\right) \in X\left(\sqrt{c / q_{1}}, \ldots, \sqrt{c / q_{t}} ; N / N^{\prime}\right)$. Lemma 3 implies

$$
\begin{aligned}
\left|S \bmod N / N^{\prime}\right| & \leq\left|X\left(\sqrt{c / q_{1}}, \ldots, \sqrt{c / q_{t}} ; N / N^{\prime}\right)\right| \\
& <3^{t}\left(N / N^{\prime}\right) \Pi \max \left(\sqrt{c / q_{i}} \cdot N / N^{\prime}, 1\right),
\end{aligned}
$$

which contradicts, the assumption.

Theorem. Let $q_{1}, \ldots, q_{t}$ be positive numbers, $r_{1}, \ldots, r_{t}$ non-zero integers with $r_{1}$ $=1$, and $N$ a natural number. Then we have

$$
\begin{aligned}
K & :=\min _{b \in \mathbf{Z}, N \times b}\left(\sum_{j=1}^{t}\left\lceil b r_{j} / N\right\rceil^{2} q_{j}\right) \\
& \geq \min \left(\left(\frac{r_{1}}{2 r_{2}}\right)^{2} q_{1}, \ldots,\left(\frac{r_{t-1}}{2 r_{t}}\right)^{2} q_{t-1}, N^{-2} \sum_{j=1}^{t} r_{j}^{2} q_{j}\right) .
\end{aligned}
$$

Proof. Suppose that 


$$
K \leq\left(\frac{r_{j}}{2 r_{j+1}}\right)^{2} q_{j} \text { for } j=1, \ldots, t-1
$$

We will show that $K$ is attained at $b=1$. Suppose that an integer $b$ gives the minimum $K$ and $|b| \leq N / 2$. The condition $N \times b$ implies $b \neq 0$. First, we claim

$$
\left|b r_{j}\right| \leq N / 2 \text { for } j=1, \ldots, t .
$$

When $j=1$, it is true because of $r_{1}=1$. Suppose that (2) is true for $j=i$ ( $\leq t-$ $1)$; then we have $\left|b r_{i}\right| \leq N / 2$ and hence $K \geq\left\lceil b r_{i} / N\right\rceil^{2} q_{i}=\left(b r_{i} / N\right)^{2} q_{i}$, which yields $|b| \leq \sqrt{K / q_{i}} \cdot N /\left|r_{i}\right|$. Now using (1), we have $\left|b r_{i+1}\right| \leq \sqrt{K / q_{i}} \cdot N /\left|r_{i}\right| \cdot$ $\left|r_{i+1}\right| \leq\left|r_{\imath}\right| /\left(2\left|r_{i+1}\right|\right) \cdot N /\left|r_{i}\right| \cdot\left|r_{i+1}\right|=N / 2$. Thus (2) has been shown inductively.

The condition (2) implies $\left\lceil b r_{j} / N\right\rceil^{2}=\left(b r_{j} / N\right)^{2}$ and then

$$
K=\sum_{j=1}^{t}\left(b r_{j} / N\right)^{2} q_{j}=b^{2} / N^{2} \sum_{j=1}^{t} r_{j}^{2} q_{j} \geq N^{-2} \sum_{j=1}^{t} r_{j}^{2} q_{j}
$$

where the equality occurs for $b= \pm 1$. This completes the proof.

Corollary 1. Let $q_{j}, r_{j}, N, K$ be those in Theorem, and put

$$
\Delta:=\prod_{k=1}^{t} q_{k}, \quad \Delta_{j}:=\Delta^{-(j-1) / t} \prod_{k<j} q_{k}, \quad \eta_{j}:=\frac{\left|r_{j}\right|}{N^{(j-1) / t} \Delta_{j}^{1 / 2}}
$$

for $j=1, \ldots, t$. Then we have

(i) $\quad 4\left(\frac{\Delta}{N^{2}}\right)^{-1 / t} K$

$$
\begin{aligned}
& \geq \min \left(\left(\eta_{1} / \eta_{2}\right)^{2}, \ldots,\left(\eta_{t-1} / \eta_{t}\right)^{2}, \sum_{j=1}^{t} \eta_{j}^{2}\left(\Delta / N^{2}\right)^{1-j / t}\left(\prod_{j<k \leq t} q_{k}\right)^{-1}\right) \\
& \geq \min \left(\left(\eta_{1} / \eta_{2}\right)^{2}, \ldots,\left(\eta_{t-1} / \eta_{t}\right)^{2}, \eta_{t}^{2}\right)
\end{aligned}
$$

(ii) $\eta_{1}=1$,

(iii) if $q_{1} \geq q_{2} \geq \cdots \geq q_{t}$, then we have $\Delta, \geq 1$ for $j=1, \ldots, t$.

Proof. $\eta_{1}=1$ is trivial. We have for $j<t$,

$$
\begin{aligned}
\left(\frac{r_{j}}{r_{j+1}}\right)^{2} q_{j} & =\frac{\eta_{j}^{2} N^{2(j-1) / t} \Pi_{k<j} q_{k} \cdot \Delta^{-(j-1) / t}}{\eta_{j+1}^{2} N^{2 j / t} \Pi_{k<j+1} q_{k} \cdot \Delta^{-j / t}} q_{j} \\
& =\left(\frac{\eta_{j}}{\eta_{j+1}}\right)^{2}\left(\frac{\Delta}{N^{2}}\right)^{1 / t},
\end{aligned}
$$


and hence

$$
\left(\frac{r_{j}}{r_{t}}\right)^{2} q_{j} \cdots q_{t-1}=\left(\frac{\eta_{j}}{\eta_{t}}\right)^{2}\left(\frac{\Delta}{N^{2}}\right)^{(t-j) / t},
$$

and then by putting $j=1$

$$
\frac{r_{t}^{2}}{N^{2}} q_{t}=\eta_{t}^{2}\left(\Delta / N^{2}\right)^{1 / t}
$$

Therefore we have

$$
\left(\frac{r_{j}}{N}\right)^{2} q_{j}=\eta_{j}^{2}\left(\Delta / N^{2}\right)^{1-(j-1) / t}\left(\prod_{j<k \leq t} q_{k}\right)^{-1}
$$

The inequality in (i) follows trivially from the above.

Suppose $q_{1} \geq q_{2} \geq \cdots \geq q_{t}$; then we have

$$
\begin{aligned}
\Delta_{j}=\prod_{k<j} q_{k} \cdot \Delta^{-(j-1) / t} & =\prod_{k<j} q_{k}^{1-(j-1) / t} \cdot \prod_{k \geq j} q_{k}^{-(j-1) / t} \\
& \geq q_{j}^{\Sigma_{k<j}(1-(j-1) / t)} \cdot q_{j}^{\Sigma_{k \geq j}-(j-1) / t}=1 .
\end{aligned}
$$

Corollary 2. Suppose $t=2$ in Theorem. Then we have

$$
K \gg \sqrt{q_{1} q_{2}} / N \text { if } r_{2}^{2} \asymp \sqrt{q_{1} / q_{2}} N \text { or if both }\left(r_{2}, N\right)=1 \text { and } \sqrt{q_{1} / q_{2}} N \ll 1 \text {. }
$$

Proof. It follows from Theorem that

$$
4\left(\frac{q_{1} q_{2}}{N^{2}}\right)^{-1 / 2} K \geq \min \left(\frac{\sqrt{q_{1} / q_{2}} N}{r_{2}^{2}}, \frac{r_{2}^{2}}{\sqrt{q_{1} / q_{2}} N}+\frac{1}{\sqrt{q_{2} / q_{1}} N}\right) .
$$

Hence the first assertion is clear. Next we assume $\left(r_{2}, N\right)=1$ and take an integer $R_{2}$ such that $r_{1} \equiv R_{2} r_{2} \bmod N$ and $0<R_{2}<N$, and we note that $B:=b r_{2}$ runs over the same set $\bmod N$ as $b$. Interchanging the suffices 1 and 2 , we have

$$
4\left(\frac{q_{1} q_{2}}{N^{2}}\right)^{-1 / 2} K \geq \min \left(\frac{\sqrt{q_{2} / q_{1}} N}{R_{2}^{2}}, \frac{R_{2}^{2}}{\sqrt{q_{2} / q_{1}} N}+\frac{1}{\sqrt{q_{1} / q_{2}} N}\right) .
$$

The second assertion follows from $\sqrt{q_{2} / q_{1}} N R_{2}^{-2} \geq\left(\sqrt{q_{1} / q_{2}} N\right)^{-1} \gg 1$.

Corollary 3. Let $q_{1}, \ldots, q_{t}$ be positive numbers and $N(>2)$ a natural number. Let $x_{1}, \ldots, x_{t}, x$ be integers and suppose that one of $2 x_{1}, \ldots, 2 x_{t}, 2 x$ is not congruent to $0 \bmod N$. Then there are integers $r_{1}, \ldots, r_{t}$ such that $\sum_{i=1}^{t} r_{i} x_{i} \not \equiv x \bmod N$ and 


$$
\min _{b \in \mathbf{Z}, N \nmid b}\left(\sum_{i=1}^{t}\left\lceil b r_{i} / N\right\rceil^{2} q_{i}\right) \gg\left(N^{-2} \prod_{i=1}^{t} q_{i}\right)^{1 / t}
$$

Proof. We may suppose $q_{1} \geq q_{2} \geq \cdots \geq q_{t}$. For integers $r_{1}= \pm 1, r_{2}, \ldots$, $r_{t}$, let $\Delta, \Delta_{i}, \eta_{i}$ be those in Corollary 1 . By virtue of $\Delta_{i} \geq 1$, we can choose $r_{i}$ so that $\eta_{i} \asymp 1$ for $i>1$. We note that this property also holds for $r_{i}+1$ instead of $r_{i}$ because of $\Delta_{i} \geq 1$. If $\sum_{i=1}^{t} r_{i} x_{i} \not \equiv x \bmod N$, then Corollary 1 implies the assertion. Suppose

$$
\sum_{i=1}^{t} R_{i} x_{i} \equiv x \bmod N \text { for } R_{1}= \pm 1, R_{i}=r_{i}, r_{i}+1(i>1) .
$$

Substituting $R_{j}=r_{j}, r_{j}+1$, we have $x_{j} \equiv 0 \bmod N$ for $j>1$. Hence we have $x_{1}$ $\equiv-x_{1} \equiv x \bmod N$, and then $2 x \equiv 2 x_{1} \equiv 0 \bmod N$. This is the contradiction.

Proposition 2. Let $q_{1}, \ldots, q_{t}$ be positive numbers, $r_{1}, \ldots, r_{t}$ integers, and $N a$ natural number with $\left(r_{1}, \ldots, r_{t}, N\right)=1$. Put

$$
\Delta=\prod_{i=1}^{t} q_{i}, \quad K:=\min _{b \in \mathbf{Z}, N{ }^{\prime}}\left(\sum_{j=1}^{t}\left\lceil b r_{i} / N\right\rceil^{2} q_{j}\right) .
$$

Then we have

$$
K \geq \min \left\{q_{1}, \ldots, q_{t}\right\} \text { or } K \ll_{t}\left(\Delta / N^{2}\right)^{1 / t}
$$

Proof. Define a positive lattice $M:=\mathbf{Z}\left[v_{1}, \ldots, v_{t}\right]$ by $\left(B\left(v_{i}, v_{j}\right)\right)=\operatorname{diag}\left(q_{1}\right.$, $\left.\ldots, q_{t}\right)$ and put $M^{\prime}:=M+\mathbf{Z}\left[\left(\sum r_{i} v_{i}\right) / N\right]$. Then we have $\left[M^{\prime}: M\right]=N$ and hence $\mathrm{d} M=N^{2} \mathrm{~d} M^{\prime}$. The general theory of positive definite quadratic forms implies $\min \left(M^{\prime}\right) \ll_{t}\left(\mathrm{~d} M^{\prime}\right)^{1 / t}=\left(\Delta / N^{2}\right)^{1 / t}$. On the other hand, Lemma 1 implies $\min \left(M^{\prime}\right) \asymp_{t} \min (\min (M), K)$, and hence if $K<\min (M)=\min \left\{q_{1}, \ldots, q_{t}\right\}$, we have $K \asymp_{t} \min \left(M^{\prime}\right) \ll_{t}\left(\mathrm{~d} M^{\prime}\right)^{1 / t}=\left(\Delta / N^{2}\right)^{1 / t}$.

Example. In Proposition 2, put $t=2, r_{1}=r_{2}=1, q_{1}=1, q_{2}=N^{2+\varepsilon}(\varepsilon>$ $0)$. Then we have

$$
K=N^{-2}+N^{\varepsilon},\left(\Delta / N^{2}\right)^{1 / t}=N^{\varepsilon / 2} .
$$

Hence $K \ll_{t}\left(\Delta / N^{2}\right)^{1 / t}$ is false in this case.

Proposition 3. Let $t, q_{i}, r_{i}, N, \Delta, K$ be those in Proposition 2. Then there is a positive number $\delta_{t}$ dependent on $t$ such that 


$$
K \ll_{t}\left(\Delta / N^{2}\right)^{1 / t} \text { if }\left(\Delta / N^{2}\right)^{1 / t}<\delta_{t} \min \left\{q_{1}, \ldots, q_{t}\right\}
$$

Proof. We use induction on $t$. The assertion is clearly true for $t=1$, since $K$ $=\Delta / N^{2}$. We may suppose $q_{1} \leq \cdots \leq q_{t}$ without loss of generality. Put $M=$ $\left(r_{2}, \ldots, r_{t}, N\right)$. First, suppose $M \neq 1$; then for $b:=N / M(\neq 0 \bmod N), b r_{1} / N$ is not an integer and therefore we have

$$
K \leq \sum_{i=1}^{t}\left\lceil b r_{i} / N\right\rceil^{2} q_{i}=\left\lceil b r_{1} / N\right\rceil^{2} q_{1} \leq q_{1} / 4
$$

which implies $K \ll_{t}\left(\Delta / N^{2}\right)^{1 / t}$ by virtue of Proposition 2.

Hereafter we suppose $M=1$. We choose a sufficiently small constant $\delta_{t}$. By the assumption, we have $\Delta / N^{2}<\delta_{t}^{t} q_{1}^{t} \leq \delta_{t}^{t} q_{1} q_{2}^{t-1}$ and hence $q_{2} \cdots q_{t} / N^{2}$ $<\delta_{t}^{t} q_{2}^{t-1}<\delta_{t-1}^{t-1} q_{2}^{t-1}$ if $\delta_{t}^{t}<\delta_{t-1}^{t-1}$. Then the induction hypothesis implies

$$
\min _{b \in \mathbf{Z}, N \chi b} \sum_{i=2}^{t}\left\lceil b r_{i} / N\right\rceil^{2} q_{i}<c_{t-1}\left(q_{2} \cdots q_{t} / N^{2}\right)^{1 /(t-1)}
$$

for some constant $c_{t-1}$. Therefore, for the integer $b$ which gives the minimum of the left-hand side in the above inequality, we have

$$
K \leq \sum_{i=1}^{t}\left\lceil b r_{i} / N\right\rceil^{2} q_{i} \leq q_{1} / 4+c_{t-1}\left(q_{2} \cdots q_{t} / N^{2}\right)^{1 /(t-1)}
$$

Here we have

$$
\left(q_{2} \cdots q_{t} / N^{2}\right)^{1 /(t-1)}=q_{1}^{-1 /(t-1)}\left(\Delta / N^{2}\right)^{1 /(t-1)}<q_{1}^{-1 /(t-1)}\left(\delta_{t} q_{1}\right)^{t /(t-1)}=\delta_{t}^{t /(t-1)} q_{1}
$$

and hence $K \leq\left(1 / 4+c_{t-1} \delta_{t}^{t /(t-1)}\right) q_{1}<q_{1}$ if $1 / 4+c_{t-1} \delta_{t}^{t /(t-1)}<1$. Proposition 2 implies $K \ll_{t}\left(\Delta / N^{2}\right)^{1 / t}$, which completes the proof.

Proposition 4. Let $t, q_{i}, r_{i}, N, K$ be those in Proposition 2. If $N \gg_{t} 1$, then we have

$$
K \ll_{t} N^{-2 / t} \max _{i} q_{i}
$$

Proof. If $N \gg_{t} 1$, then $\min _{N \chi b} \sum_{i=1}^{t}\left\lceil b r_{i} / N\right\rceil^{2} \ll_{t} N^{-2 / t}$ follows from Proposition 3. Thus there is an integer $b \neq \equiv 0 \bmod N$ such that $\sum_{i=1}^{t}\left\lceil b r_{i} / N\right\rceil^{2} \ll{ }_{t} N^{-2 / t}$, and hence we have $K \leq \sum_{i=1}^{t}\left\lceil b r_{i} / N\right\rceil^{2} q_{i} \ll{ }_{t} N^{-2 / t} \max _{i} q_{i}$.

Proposition 5. Let $t$ be a natural number, $p$ a prime number and $r_{i}=R_{i} p^{e_{i}}$ integers with $\left(p, R_{\imath}\right)=1$ for $i=1,2, \ldots, t$. We assume that $e_{1}=0 \leq e_{2} \leq e_{3} \leq \cdots$ 
$\leq e_{t}$ and define a sequence of integers $v_{0}:=1<v_{1}<v_{2}<\cdots<v_{k}<v_{k+1}:=t+$ 1 by

$$
\begin{aligned}
& e_{v_{0}}=\cdots=e_{v_{1}-1} \\
&<e_{v_{1}}=\cdots=e_{v_{2}-1} \\
&<\quad \cdots \\
&<e_{v_{k}}= \cdots=e_{v_{v^{+1}-1}} .
\end{aligned}
$$

For a natural number $e_{t+1}\left(\geq e_{t}\right)$ and positive numbers $q_{1}, q_{2}, \ldots, q_{t}$, we put

$$
\begin{aligned}
& K:=\min _{b \in \mathbf{Z}, N \times b}\left(\sum_{j=1}^{t}\left\lceil b r_{j} / N\right\rceil^{2} q_{j}\right) \text { where } N:=p^{e_{t+1}} \\
& K_{j}:=\min _{b \in \mathbf{Z}, p^{E_{j}} \backslash b}\left(\sum_{i<v_{j}}\left\lceil b R_{i} / p^{E_{j}}\right\rceil^{2} q_{i} p^{-2\left(e_{v_{j-1}}-e_{i}\right)}\right) \text { for } j=1, \ldots, k+1
\end{aligned}
$$

where $E_{j}:=e_{v_{j}}-e_{v_{j-1}}$. Then we have $K \geq \min \left\{K_{1}, \ldots, K_{k+1}\right\}$.

Proof. Putting $v:=v_{1}, e:=e_{v_{1}}, s=e_{t+1}$, we claim that

$$
K \geq \min \left\{K_{1}, \min _{b \in \mathbf{Z}, p^{s-e} \chi b}\left(\sum_{i<v}\left\lceil b r_{i} / p^{s-e}\right\rceil^{2} q_{i} p^{-2 e}+\sum_{i \geq v}\left\lceil b r_{i} p^{-e} / p^{s-e}\right\rceil^{2} q_{i}\right)\right\} .
$$

Let us show the claim. For an integer $c$, we put

$$
K(c):=\min _{b} \sum_{i=1}^{t}\left\lceil b r_{i} / p^{s}\right\rceil^{2} q_{i}
$$

where $b$ runs over the set of integers satisfying $b \equiv c \bmod p^{s-e}$ and $b \not \equiv 0 \bmod p^{s}$. It is easy to see

$$
K(0)=\min _{B \in \mathbf{Z}, p^{e} \times B} \sum_{i<v}\left\lceil B r_{\imath} / p^{e}\right\rceil^{2} q_{\imath}=K_{1} .
$$

Next, for an integer $c\left(\not \equiv 0 \bmod p^{s-e}\right)$ we assume $K(c)$ is attained at $b(\equiv c \bmod$ $\left.p^{s-e}\right)$. Then we have

$$
K(c)=\sum_{i<v}\left\lceil b r_{i} / p^{s}\right\rceil^{2} q_{i}+\sum_{i \geq v}\left\lceil c r_{i} / p^{s}\right\rceil^{2} q_{i}
$$

Now we show

$$
\left|\left\lceil b r_{i} p^{-s}\right\rceil\right| \geq\left|\left\lceil b r_{i} p^{-(s-e)}\right\rceil\right| p^{-e} \text { for } i<v \text {. }
$$

We define integers $B, B_{1}, B_{2}$ by

$$
B \equiv b r_{i} \bmod p^{s},-p^{s} / 2 \leq B<p^{s} / 2,
$$




$$
B=B_{1}+B_{2} p^{s-e},-p^{s-e} / 2 \leq B_{1}<p^{s-e} / 2 .
$$

We have only to show $\left|B / p^{s}\right| \geq\left|B_{1} / p^{s-e}\right| p^{-e}$, and may assume $B \geq 0$ without loss of generality. If $0 \leq B_{1}<p^{s-e} / 2$, then we have $B_{2} \geq 0$ and then $B / p^{s}=$ $B_{1} / p^{s}+B_{2} / p^{e} \geq B_{1} / p^{s}=\left(B_{1} / p^{s-e}\right) p^{-e}$, which is the required inequality. If $-p^{s-e} / 2 \leq B_{1}<0$, then we have $B_{2}>0$ and hence $B / p^{s}=\left(B_{1}+p^{s-e}+\left(B_{2}\right.\right.$ $\left.-1) p^{s-e}\right) / p^{s} \geq\left(B_{1}+p^{s-e}\right) / p^{s}=\left(B_{1} / p^{s-e}+1\right) p^{-e} \geq\left|B_{1} / p^{s-e}\right| p^{-e}$, because of $x+1 \geq|x|$ for a real number $x:=B_{1} / p^{s-e}$ in $[-1 / 2,0)$. Thus we have shown the inequality (2) and

$$
\begin{aligned}
K(c) & \geq \sum_{i<v}\left\lceil b r_{i} / p^{s-e}\right\rceil^{2} q_{i} p^{-2 e}+\sum_{i \geq v}\left\lceil c r_{i} / p^{s}\right\rceil^{2} q_{i} \\
& =\sum_{i<v}\left\lceil c r_{i} / p^{s-e}\right\rceil^{2} q_{\imath} p^{-2 e}+\sum_{i \geq v}\left\lceil c r_{i} / p^{s}\right\rceil^{2} q_{i} .
\end{aligned}
$$

Hence the identity $K=\min \{K(c) \mid c \in \mathbf{Z}\}$ implies

$$
K \geq \min \left\{K(0), \min _{c \neq 0 \bmod p^{s-e}}\left(\sum_{i<v}\left\lceil c r_{i} / p^{s-e}\right\rceil^{2} q_{i} p^{-2 e}+\sum_{i \geq v}\left\lceil c r_{i} / p^{s}\right\rceil^{2} q_{i}\right)\right\}
$$

implies the inequality (1).

Now the assertion of the lemma is shown by induction on $k$. By the claim (1), we have $K \geq \min \left\{K_{1}, K^{\prime}\right\}$, and

$$
K^{\prime}:=\min _{b \in \mathbf{Z}, N^{\prime} \rtimes b}\left(\sum_{i<v_{1}}\left\lceil b R_{i} / N^{\prime}\right\rceil^{2} q_{i} p^{-2 e_{v_{1}}}+\sum_{i \geq v_{1}}\left\lceil b r_{i} p^{-e_{v_{1}}} / N^{\prime}\right\rceil^{2} q_{i}\right)
$$

where $N^{\prime}:=p^{s-e_{v_{1}}}$. Put

$$
\begin{aligned}
& V_{\imath}:=v_{i+1} \text { for } i=1, \ldots, k-1, \text { and } V_{0}:=1, V_{k}:=t+1, \\
& e_{i}^{\prime}:=\left\{\begin{array}{ll}
0 & \text { if } i<v_{1}, \\
e_{i}-e_{v_{1}} & \text { if } i \geq v_{1},
\end{array} \quad Q_{i}:= \begin{cases}q_{i} p^{-2 e_{v_{1}}} & \text { if } i<v_{1}, \\
q_{i} & \text { if } i \geq v_{1} .\end{cases} \right.
\end{aligned}
$$

Then we have

$$
\begin{gathered}
e_{v_{j}}^{\prime}-e_{v_{j-1}}^{\prime}=e_{v_{j+1}}-e_{v_{j}} \text { for } j=1, \ldots, k \\
Q_{i} p^{-2\left(e_{v_{j-1}}^{\prime}-e_{i}^{\prime}\right)}=q_{i} p^{-2\left(e_{v_{j}}-e_{i}\right)} \text { for } i<v_{j}(j=1, \ldots, k) .
\end{gathered}
$$

Therefore we can apply the induction hypothesis to $K^{\prime}$.

\section{Distribution of isotropic vectors}

In this section, we study the distribution of isotropic vectors in a quadratic space over a finite prime field. $p$ denotes an odd prime number and $F_{p}$ stands for 
the prime field $\mathbf{Z} / p \mathbf{Z}$ through this section.

THEOREM 1. Let $V=F_{p}\left[e_{1}, e_{2}\right]$ be a regular quadratic space over $F_{p}$ with quadratic form $Q$. Then for every positive integer $H<p$, we have

$$
\left|\sum_{1 \leq x \leq H} \chi\left(Q\left(x e_{1}+e_{2}\right)\right)\right| \leq 2 \sqrt{p} \log p+1
$$

where $\chi$ stands for the quadratic residue symbol with $\chi(0)=0$.

To prove this, we prepare several lemmas.

Lemma 1. Let $H$ be an integer such that $1 \leq H<p$. For a function $c(x)$ on $F_{p}$ defined by

$$
c(x):= \begin{cases}1 & \text { if } 1 \leq x \leq H \\ 0 & \text { otherwise }\end{cases}
$$

we put

$$
h(y):=p^{-1} \sum_{x \in F_{p}} c(z) e(-y z / p)
$$

where $e(x)$ denotes $\exp (2 \pi i x)$. Then we have

$$
c(x)=\sum_{y \in F_{p}} h(y) e(x y / p) .
$$

Proof. The assertion follows from

$$
\begin{aligned}
& \sum_{y \in F_{p}} h(y) e(x y / p)=p^{-1} \sum_{y \in F_{p}} \sum_{z \in F_{p}} c(z) e((-y z+x y) / p) \\
= & p^{-1} \sum_{z \in F_{p}} c(z) \sum_{y \in F_{p}} c(y(x-z) / p)=c(x) .
\end{aligned}
$$

Lemma 2. For $a, b \in F_{p}$ with $a^{2}-4 b \neq 0$, let us define the function $\phi(x)$ on $F_{p}$ by $\phi(x):=x^{2}+a x+b$. Then we have

$$
\sum_{x \in F_{p}} \chi(\phi(x))=-1
$$

where $\chi$ stands for the quadratic residue symbol with $\chi(0)=0$.

Proof. See Theorem 8.2 in [1]. 
Lemma 3. For the above functions $\chi$ and $\phi$, we have

$$
\left|\sum_{x \in F_{p}} \chi(\phi(x)) e(x y / p)\right| \leq 2 \sqrt{p}
$$

Proof. We use Theorem 2G on p. 45 in [5]. We put $f(x):=\phi(x)$ and $g(x):=x$ there. Then $Y^{2}-f(X)$ is absolutely irreducible because of $\phi(x)=$ $(x+a / 2)^{2}+b-a^{2} / 4$ and $b-a^{2} / 4 \neq 0$ in $F_{p}$, and so is $Z^{p}-Z-g(X)$ by Theorem $1 \mathrm{~B}$ on p. 92 in [5]. Hence the condition (ii) in Theorem $2 \mathrm{G}$ is satisfied and we have the assertion.

Lemma 4. For the function $h(x)$ in Lemma 1, we have

$$
\sum_{y \in F_{p}^{\times}}|h(y)| \leq \log p
$$

Proof. Since $\sum_{y \in F_{p}^{\times}}|h(y)|=p^{-1} \sum_{y \in F_{p}^{\times}}\left|\sum_{1 \leq z \leq H} e(-y z / p)\right|$, the inequality on p. 56 in [6] gives the required one.

Lemma 5. Let $H$ be an integer such that $1 \leq H<p$, the functions $\chi$ and $\phi$ as above. Then putting

$$
\Phi:=\sum_{1 \leq x \leq H} \chi(\phi(x))
$$

we have

$$
|\Phi| \leq 2 \sqrt{p} \log p+1 \text {. }
$$

Proof. It is easy to see, using the function $c(x)$ and $h(x)$ in Lemma 1

$$
\begin{aligned}
\Phi & =\sum_{y \in F_{p}} \chi(\phi(x)) c(x) \\
& =\sum_{x \in F_{p}} \chi(\phi(x)) \sum_{y \in F_{p}} h(y) e(x y / p) \\
& =\sum_{x \in F_{p}} \chi(\phi(x)) h(0)+\sum_{y \in F_{p}^{\times}} h(y) \sum_{x \in F_{p}} \chi(\phi(x)) e(x y / p) \\
& =-p^{-1} \sum_{z \in F_{p}} c(z)+\sum_{y \in F_{p}^{\times}} h(y) \sum_{x \in F_{p}} \chi(\phi(x)) e(x y / p) \\
& =-H / p+\sum_{y \in F_{p}^{\times}} h(y)\left\{\sum_{x \in F_{p}} \chi(\phi(x)) e(x y / p)\right\} .
\end{aligned}
$$

Hence we have

$$
|\Phi| \leq H / p+\sum_{y \in F_{p}^{\times}}|h(y)| \cdot 2 \sqrt{p} \leq H / p+2 \sqrt{p} \log p \leq 2 \sqrt{p} \log p+1 .
$$


Proof of Theorem 1. Putting $\phi(x):=Q\left(x e_{1}+e_{2}\right)$, we show

$$
\left|\sum_{1 \leq x \leq H} \chi(\phi(x))\right| \leq 2 \sqrt{p} \log p+1
$$

If $Q\left(e_{1}\right) \neq 0$, then we can apply Lemma 5 because of $\phi(x)=Q\left(e_{1}\right)\left\{x^{2}+2 B\left(e_{1}\right.\right.$, $\left.\left.e_{2}\right) Q\left(e_{1}\right)^{-1} x+Q\left(e_{2}\right) Q\left(e_{1}\right)^{-1}\right\}$, where the bilinear form $B(x, y)$ is defined by $2 B(x, y):=Q(x+y)-Q(x)-Q(y)$. If $Q\left(e_{1}\right)=0$, then we have $B\left(e_{1}, e_{2}\right) \neq 0$ and $\phi(x)=2 B\left(e_{1}, e_{2}\right)\left(x+Q\left(e_{2}\right) /\left(2 B\left(e_{1}, e_{2}\right)\right)\right)$, and then Pólya-Vinogradov's inequality (Problem $\alpha$ ) in b. on p. 102 in [6]) yields the inequality.

Theorem 2. Let $V=F_{p}\left[e_{1}, \ldots, e_{m}\right](m \geq 3)$ be a quadratic space over $F_{p}$. Then we have the following assertions:

(i) Suppose that $Q\left(e_{i}\right)=0, B\left(e_{i}, e_{j}\right) \neq 0$ for some $i, j(i \neq j)$. Then for any $x_{k} \in F_{p}$ $(k \neq i, j)$, there are elements $y_{i} \in F_{p}, y_{j}= \pm 1$ and $u \in V$ so that

$$
v:=y_{i} e_{\imath}+y_{j} e_{j}+\sum_{k \neq \imath, j} x_{k} e_{k}
$$

is isotropic and $B(u, v) \neq 0$.

(ii) Suppose $m \geq 4$ and $\operatorname{dim} \operatorname{Rad} V \leq m-3$. Then there exists a subset $T=\left\{t_{1}, t_{2}\right.$, $\left.t_{3}\right\} \subset\{1,2, \ldots, m\}$ which satisfies the following property:

Let $S_{1}, S_{2}$ be subsets of $F_{p}$ and assume that $\left|S_{1}\right|=3$ and $S_{2}$ is a set of consecutive integers. If $p>5$ and $\left|S_{2}\right|>5 \sqrt{p} \log p$, then there are elements $x_{1}= \pm 1, x_{2} \in S_{1}, x_{3} \in S_{2}, y_{i} \in F_{p}$ for $i \notin T$ and $u \in V$ such that

$$
v=\sum_{j=1}^{3} x_{j} e_{t_{j}}+\sum_{i \notin T} y_{i} e_{i}
$$

is isotropic and $B(u, v) \neq 0$.

Proof of (i). Suppose that $Q\left(e_{i}\right)=0, B\left(e_{i}, e_{j}\right) \neq 0$ for some $i, j(i \neq j)$ and $x_{k}(k \neq i, j)$ is given. Putting $v:=y_{i} e_{i}+y_{j} e_{j}+\sum_{k \neq i, j} x_{k} e_{k}$, we have

$$
\begin{aligned}
Q(v) & =2 y_{i} B\left(e_{i}, y_{j} e_{j}+\sum_{k \neq \imath, j} x_{k} e_{k}\right)+Q\left(y_{j} e_{j}+\sum_{k \neq \imath, j} x_{k} e_{k}\right) \\
& =2 y_{i}\left(y_{j} B\left(e_{i}, e_{j}\right)+B\left(e_{i}, \sum_{k \neq \imath, j} x_{k} e_{k}\right)\right)+Q\left(y_{j} e_{j}+\sum_{k \neq i, j} x_{k} e_{k}\right) .
\end{aligned}
$$

Because of $B\left(e_{i}, e_{j}\right) \neq 0$, we can take $y_{j}= \pm 1$ so that $y_{j} B\left(e_{i}, e_{j}\right)+B\left(e_{i}, \sum_{k \neq i, j}\right.$ $\left.x_{k} e_{k}\right) \neq 0$ and then we can choose $y_{i}$ so that $v$ is isotropic. For $u:=e_{i}$, we have

$$
B(u, v)=y_{j} B\left(e_{j}, e_{j}\right)+B\left(e_{i}, \sum_{k \neq i, j} x_{k} e_{k}\right) \neq 0 .
$$


To prove the assertion (ii), we prepare two lemmas.

Lemma 6. Let $W=F_{p}\left[w_{1}, \ldots, w_{n}\right](n \geq 3)$ be a quadratic space over $F_{p}$ and assume that $Q\left(w_{1}\right) \neq 0$, and $\operatorname{dim} \operatorname{Rad} W \leq n-2$. For a subset $S \subset F_{p}$ with $|S|=$ 3 , there exist an element $x \in S$ and suffices $i, j>1(i \neq j)$ such that $F_{p}\left[w_{i}+x w_{j}\right.$, $w_{1}$ ] is a regular quadratic space.

Proof. Putting $w_{i}^{\prime}:=w_{\imath}-\frac{B\left(w_{1}, w_{i}\right)}{Q\left(w_{1}\right)} w_{1}$, we have a decomposition $W=$ $F_{p}\left[w_{1}\right] \perp F_{p}\left[w_{2}^{\prime}, \ldots, w_{n}^{\prime}\right]$. It is easy to see, for $i, j$

$F_{p}\left[w_{\imath}+x w_{j}, w_{1}\right]$ is not regular for any $x \in S$

$\Leftrightarrow Q\left(w_{i}+x w_{j}\right) Q\left(w_{1}\right)=B\left(w_{i}+x w_{j}, w_{1}\right)^{2}$ for any $x \in S$

$\Leftrightarrow\left(Q\left(w_{j}\right) Q\left(w_{1}\right)-B\left(w_{j}, w_{1}\right)^{2}\right) x^{2}+2\left(B\left(w_{i}, w_{j}\right) Q\left(w_{1}\right)-B\left(w_{1}, w_{i}\right) B\left(w_{1}, w_{j}\right)\right) x$

$+Q\left(w_{i}\right) Q\left(w_{1}\right)-B\left(w_{i}, w_{1}\right)^{2}=0$ for any $x \in S$

$\Leftrightarrow\left\{\begin{array}{l}Q\left(w_{k}\right) Q\left(w_{1}\right)=B\left(w_{k}, w_{1}\right)^{2} \text { for } k=j, i, \\ B\left(w_{i}, w_{j}\right) Q\left(w_{1}\right)=B\left(w_{1}, w_{i}\right) B\left(w_{1}, w_{j}\right) .\end{array}\right.$

Moreover we have

$$
\begin{aligned}
Q\left(w_{i}^{\prime}\right) & =Q\left(w_{1}\right)^{-1}\left(Q\left(w_{1}\right) Q\left(w_{\imath}\right)-B\left(w_{1}, w_{\imath}\right)^{2}\right), \\
B\left(w_{\imath}^{\prime}, w_{j}^{\prime}\right) & =Q\left(w_{1}\right)^{-1}\left(Q\left(w_{1}\right) B\left(w_{i}, w_{j}\right)-B\left(w_{1}, w_{j}\right) B\left(w_{i}, w_{1}\right)\right) .
\end{aligned}
$$

Now suppose that $F_{p}\left[w_{i}+x w_{j}, w_{1}\right]$ is not regular for any $i, j>1(i \neq j)$ and for any $x \in S$. Then the above implies $Q\left(w_{i}^{\prime}\right)=B\left(w_{i}^{\prime}, w_{j}^{\prime}\right)=0$ for the above $i, j$, which implies $Q\left(F_{p}\left[w_{2}^{\prime}, \ldots, w_{n}^{\prime}\right]\right)=0$, and then contradicts $\operatorname{dim} \operatorname{Rad} W \leq n-2$.

Lemma 7. Let $W=F_{p}\left[w_{1}, \ldots, w_{n}\right](n \geq 3)$ be a quadratic space over $F_{p}$ and suppose $Q\left(w_{1}\right) \neq 0, \operatorname{dim} \operatorname{Rad} W \leq n-2$. Then we have the following:

Let $S_{1}, S_{2}$ be subsets of $F_{p}$ and assume that $\left|S_{1}\right|=3$ and $S_{2}$ is a set of consecutive integers. If $p>5$ and $\left|S_{2}\right|>5 \sqrt{p} \log p$, then there are elements $x \in$ $S_{1}, y \in S_{2}$, and indeces $i, j>1(i \neq j)$ such that $Q\left(w_{i}+x w_{j}+y w_{1}\right) \in$ $\left(F_{p}^{\times}\right)^{2}$.

Proof. By virtue of Lemma 6, there exist suffices $i, j>1(i \neq j)$ and $x \in S_{1}$ such that $F_{p}\left[w_{i}+x w_{j}, w_{1}\right]$ is regular. Suppose $Q\left(w_{i}+x w_{j}+y w_{1}\right) \notin\left(F_{p}^{\times}\right)^{2}$ for any $y \in S_{2}$. By putting $t:=\left|\left\{y \in S_{2} \mid Q\left(w_{i}+x w_{j}+y w_{1}\right)=0\right\}\right|, Q\left(w_{1}\right) \neq 0$ yields $0 \leq t \leq 2$ and the supposition implies $\sum_{y \in S_{2}} \chi\left(Q\left(w_{i}+x w_{j}+y w_{1}\right)\right)=$ $-\left(\left|S_{2}\right|-t\right)$, where $\chi$ denotes the quadratic residue symbol. Theorem 1 yields 
$\left|\sum_{y \in S_{2}} \chi\left(Q\left(w_{i}+x w_{j}+y w_{1}\right)\right)\right| \leq 2(2 \sqrt{p} \log p+1)$, and hence we have $\left|S_{2}\right|$ $\leq 2(2 \sqrt{p} \log p+1)+2$. If $\left|S_{2}\right|>5 \sqrt{p} \log p$, which yields $p=3$ or 5 .

Proof of (ii) in Theorem 2. First, suppose that $Q\left(e_{i}\right)=0$ for every $i$; then the assumption $\operatorname{dim} \operatorname{Rad} V \leq m-3$ yields that there are indeces $i, j(i \neq j)$ such that $B\left(e_{i}, e_{j}\right) \neq 0$. Let $T$ be a set $\left\{t_{1}, t_{2}, t_{3}\right\}$ with $t_{1}=j$ and $i \notin T$. For $x_{2} \in S_{1}, x_{3}$ $\in S_{2}$, the assertion (i) implies that $y e_{i}+x_{1} e_{t_{1}}+x_{2} e_{t_{2}}+x_{3} e_{t_{3}}$ for some $y \in F_{p}$ and $x_{1}= \pm 1$ is a required element.

Next suppose that $Q\left(e_{i}\right) \neq 0$ for some index $i$. For simplicity, we may assume $i=1$ :

$$
Q\left(e_{1}\right) \neq 0
$$

and put

$$
w_{i}:=e_{\imath}-\frac{B\left(e_{i}, e_{1}\right)}{Q\left(e_{1}\right)} e_{1} \text {. }
$$

Putting $W=F_{p}\left[w_{2}, \ldots, w_{m}\right]$, we have $V=F_{p}\left[e_{1}\right] \perp W$ and $\operatorname{dim} \operatorname{Rad} W=$ $\operatorname{dim} \operatorname{Rad} V \leq m-3=\operatorname{dim} W-2$. We note that for an element $v:=\sum_{i=1}^{m} x_{i} e_{i} \in V$,

$$
\left\{\begin{array}{l}
v \quad=\sum_{i=1}^{m} x_{i}\left(w_{i}+\frac{B\left(e_{i}, e_{1}\right)}{Q\left(e_{1}\right)} e_{1}\right)=Q\left(e_{1}\right)^{-1} B\left(e_{1}, v\right) e_{1}+\sum_{i=2}^{m} x_{\imath} w_{\imath} \\
Q(v)=Q\left(e_{1}\right)^{-1} B\left(e_{1}, v\right)^{2}+Q\left(\sum_{i=2}^{m} x_{i} w_{i}\right)
\end{array}\right.
$$

Case (I). Suppose that there is an index $k(\geq 2)$ such that $Q\left(w_{k}\right) \neq 0$. By applying Lemma 7 to the quadratic space $W$ scaled by $-Q\left(e_{1}\right)^{-1}$, there are distinct indeces $i, j, k$ with $i, j \geq 2$ and $x_{j} \in S_{1}, x_{k} \in S_{2}$ suth that

$$
-Q\left(e_{1}\right)^{-1} Q\left(w_{i}+x_{j} w_{j}+x_{k} w_{k}\right)=r^{2}
$$

for some element $r \in F_{p}{ }^{\times}$. By putting

$$
v:=x_{1} e_{1}+x_{i} e_{i}+x_{j} e_{j}+x_{k} e_{k}
$$

for $x_{1} \in F_{p}, x_{i}=1$, (1) implies $Q(v)=Q\left(e_{1}\right)^{-1} B\left(e_{1}, v\right)^{2}-Q\left(e_{1}\right) r^{2}$. Now we choose $x_{1}$ so that $B\left(e_{1}, v\right)=\sum_{h=1, i, j, k} x_{h} B\left(e_{h}, e_{1}\right)=Q\left(e_{1}\right) r$ because of the assumption $B\left(e_{1}, e_{1}\right)=Q\left(e_{1}\right) \neq 0$. Hence we have $Q(v)=0$ and $B\left(e_{1}, v\right)=$ $Q\left(e_{1}\right) r \neq 0$ and have completed the proof of (ii) in the case of (I), by taking $t_{1}=i$, $t_{2}=j, t_{3}=k$.

Case (II). Suppose that $Q\left(w_{i}\right)=0$ if $i \geq 2$. Since $\operatorname{dim} \operatorname{Rad} W \leq \operatorname{dim} W-2$, there are indeces $i, j \geq 2(i \neq j)$ such that $B\left(w_{i}, w_{j}\right) \neq 0$. For simplicity, we may assume $B\left(w_{2}, w_{3}\right) \neq 0$. First, suppose $m \geq 5$; then put $z:=x_{2} w_{4}+x_{3} w_{5}$ for 
$x_{2} \in S_{1}, x_{3} \in S_{2} \quad$ and $\quad v^{\prime}:=y w_{2}+x_{1} w_{3}+z$ for $y, x_{1} \in F_{p}$. Since $Q\left(v^{\prime}\right)=$ $2 y\left(x_{1} B\left(w_{2}, w_{3}\right)+B\left(w_{2}, z\right)\right)+2 x_{1} B\left(w_{3}, z\right)+Q(z)$, we choose $y \in F_{p}$ and $x_{1}=$ \pm 1 so that $x_{1} B\left(w_{2}, w_{3}\right)+B\left(w_{2}, z\right) \neq 0$ and $Q\left(v^{\prime}\right)=-Q\left(e_{1}\right)$. By putting $v:=$ $x e_{1}+y e_{2}+x_{1} e_{3}+x_{2} e_{4}+x_{3} e_{5}$ for $x \in F_{p}$, we can choose $x$ so that $B\left(e_{1}, v\right)=$ $Q\left(e_{1}\right)$, and then $Q(v)=0$ follows from (1) and we complete the proof of the assertion (ii) in case of $m \geq 5$, putting $t_{1}:=3, t_{2}:=4, t_{3}:=5$ and $u:=e_{1}$.

Next suppose $m=4$. We are assuming that $Q\left(e_{1}\right) \neq 0$ and $Q\left(w_{2}\right)=$ $Q\left(w_{3}\right)=Q\left(w_{4}\right)=0$, and $B\left(w_{2}, w_{3}\right) \neq 0$. For an element $v=x_{4} e_{1}+x_{3} e_{2}+x_{1} e_{3}$ $+x_{2} e_{4} \in V$, (1) implies

$$
Q(v)=Q\left(e_{1}\right)^{-1} B\left(e_{1}, v\right)^{2}+x_{3}\left(2 x_{1} B\left(w_{2}, w_{3}\right)+2 x_{2} B\left(w_{2}, w_{4}\right)\right)+2 x_{1} x_{2} B\left(w_{3}, w_{4}\right)
$$

Suppose $x_{2} \in S_{1}$ and choose $x_{1}= \pm 1$ so that $a:=2 x_{1} B\left(w_{2}, w_{3}\right)+2 x_{2} B\left(w_{2}\right.$, $\left.w_{4}\right) \neq 0$. Now we claim that there is an element $x_{3} \in S_{2}$ so that $\chi\left(a x_{3}+\right.$ $\left.2 x_{1} x_{2} B\left(w_{3}, w_{4}\right)\right)=\chi\left(-Q\left(e_{1}\right)\right)$. If it is false, then we have

$$
\sum_{x_{3} \in S_{2}} \chi\left(a x_{3}+2 x_{1} x_{2} B\left(w_{3}, w_{4}\right)\right)=-\chi\left(-Q\left(e_{1}\right)\right)\left(\left|S_{2}\right|-t\right),
$$

where $t=\left|\left\{x_{3} \in S_{2} \mid a x_{3}+2 x_{1} x_{2} B\left(w_{3}, w_{4}\right)=0\right\}\right|=0$ or 1 . By applying Pólya-Vinogradov's inequality, we have $\left|S_{2}\right|-t \leq 2 \sqrt{p} \log p$, which contradicts $\left|S_{2}\right|>5 \sqrt{p} \log p$. Therefore there exists $x_{3} \in S_{2}$ so that $a x_{3}+2 x_{1} x_{2} B\left(w_{3}, w_{4}\right)=$ $-Q\left(e_{1}\right)^{-1} r^{2}$ for some $r \in F_{p}^{\times}$. Then we have $Q(v)=Q\left(e_{1}\right)^{-1} B\left(e_{1}, v\right)^{2}-$ $Q\left(e_{1}\right)^{-1} r^{2}$. Now we choose $x_{4}$ so that $B\left(e_{1}, v\right)=r$ because of $Q\left(e_{1}\right) \neq 0$. Then $v$ is isotropic and for $u:=w_{2}$ we have

$$
B(u, v)=x_{1} B\left(w_{2}, w_{3}\right)+x_{2} B\left(w_{2}, w_{4}\right)=a / 2 \neq 0,
$$

which completes the proof of the assertion (ii) with $t_{1}:=3, t_{2}:=4, t_{3}:=2$.

\section{Transformation matrix}

Let us give a result to combine the reduced form at the infinite prime with a Jordan decomposition at a finite prime.

THEOREM. Let $p$ be a prime number and $r, m$ positive integers with $r<m$. Let $S^{(m)}$ be a regular symmetric integral matrix and we write $S=\left(\begin{array}{cc}S_{1}^{(r)} & S_{2} \\ S_{3} & S_{4}\end{array}\right)$ and let $D_{1} \in M_{m-r}\left(\mathbf{Z}_{p}\right), D_{2} \in M_{r}\left(\mathbf{Z}_{p}\right)$ be regular matrices and suppose that $p^{t_{1}}, \ldots, p^{t_{m-r}}$ (resp. $p^{t_{m-r+1}}, \ldots, p^{t_{m}}$ ) be elementary divisors of $D_{1}$ (resp. $D_{2}$ ) over $\mathbf{Z}_{p}$ and $t_{1} \leq \ldots$ 
$\leq t_{m}$. Let $A^{(m)}=\left(\begin{array}{cc}A_{1}^{(r, m-r)} & A_{2}^{(r)} \\ A_{3}^{(m-r)} & A_{4}^{(m-r, r)}\end{array}\right)$ be an integral matrix with $\operatorname{det} A= \pm 1$.

Assume that for a natural number $e$,

$$
\begin{gathered}
A_{4} \equiv 0 \bmod p^{e}, t_{m-r}<e+t_{1} \leq \min \left(t_{m}+1, t_{m-r+1}\right) \\
S[A] \equiv\left(\begin{array}{cc}
D_{1} & 0 \\
0 & D_{2}
\end{array}\right) \bmod p^{t_{m}+1}
\end{gathered}
$$

Then $S_{4}$ and $D_{1}$ have the same elementary divisors over $\mathbf{Z}_{p}$ and $S_{3} \equiv 0 \bmod p^{e+t_{1}}$, and the matrix $S_{4}^{-1} S_{3}$ is integral over $\mathbf{Z}_{p}$ and both $S_{1}-S_{4}^{-1}\left[S_{3}\right]$ and $D_{2}$ have the same elementary divisors over $\mathbf{Z}_{p}$.

Proof. We note

$$
A^{-1} \equiv\left(\begin{array}{cc}
0^{(m-r, r)} & A_{3}^{-1} \\
A_{2}^{-1} & -A_{2}^{-1} A_{1} A_{3}^{-1}
\end{array}\right) \bmod p^{e} \mathbf{Z}_{p} .
$$

By virtue of

$$
p^{-t_{1}} S[A] \equiv p^{-t_{1}}\left(\begin{array}{cc}
D_{1} & 0 \\
0 & D_{2}
\end{array}\right) \bmod p^{t_{m}-t_{1}+1} \text { and } t_{m}-t_{1}+1 \geq e
$$

we have

$$
\begin{aligned}
S & \equiv\left(\begin{array}{cc}
D_{1} & 0 \\
0 & D_{2}
\end{array}\right)\left[\left(\begin{array}{cc}
0 & A_{3}^{-1} \\
A_{2}^{-1} & -A_{2}^{-1} A_{1} A_{3}^{-1}
\end{array}\right)\right] \bmod p^{e+t_{1}} \\
& \equiv\left(\begin{array}{cc}
0^{(r)} & 0^{(r, m-r)} \\
0^{(m-r, r)} & D_{1}\left[A_{3}^{-1}\right]
\end{array}\right) \bmod p^{e+t_{1}},
\end{aligned}
$$

by $D_{2} \equiv 0 \bmod p^{t_{m-r+1}}$. Hence $S_{4}$ and $D_{1}$ have the same elementary divisors over $\mathbf{Z}_{p}$ and we have $S_{3} \equiv 0 \bmod p^{e+t_{1}}$ and then $S_{4}^{-1} S_{3}$ is integral over $\mathbf{Z}_{p}$ by the condition $t_{m-r}<e+t_{1}$. By the identity

$$
\left.S=\left(\begin{array}{cc}
S_{1}-S_{4}^{-1}\left[S_{3}\right] & 0 \\
0 & S_{4}^{(m-r)}
\end{array}\right)\left[\begin{array}{cc}
1_{r} & 0 \\
S_{4}^{-1} S_{3} & 1_{n-r}
\end{array}\right)\right],
$$

both $D_{2}$ and $S_{1}-S_{4}^{-1}\left[S_{3}\right]$ have the same elementary divisors over $\mathbf{Z}_{p}$.

\section{Theorem}

The following is the destination of this paper. 
THEOREM. Let $m$ be an integer $\geq 6$ and $N$ a positive lattice of rank $2 m$. For a positive number $\kappa$, there is a positive number $\kappa_{1}=\kappa_{1}(\kappa, N)$ satisfying the following condition:

Let $M$ be a positive lattice of $\operatorname{rank}(M)=m$ and $\min (M)>\kappa_{1}$ and $M_{p}$ is represented by $N_{p}$ for every prime $p$. Then there is a lattice $M^{\prime} \supset M$ such that $\min \left(M^{\prime}\right)>\kappa$ and $M_{p}^{\prime}$ is primitively represented by $N_{p}$ for every prime $p$.

The rest of this section is devoted to the proof.

We fix a basis $\left\{v_{1}, \ldots, v_{m}\right\}$ of $M$ so that $\left(B\left(v_{i}, v_{j}\right)\right)$ is reduced in the sense of Minkowski, and we define a transformation matrix $A=\left(a_{i j}\right)$ by

$$
\left(w_{1}, \ldots, w_{m}\right)=\left(v_{1}, \ldots, v_{m}\right) A
$$

for another basis $\left\{w_{1}, \ldots, w_{m}\right\}$ of $M$.

Lemma 1. Let $M$ be a positive lattice such that $\operatorname{rank}(M) \geq 4, s(M) \subset p \mathbf{Z}$ and suppose that $M_{p}$ contains a p-modular sublattice of rank $\geq 3$. Then there is a positive number $\delta_{\varepsilon}$ for $0<\varepsilon<1 / 6$ satisfying the following condition:

If $p>\delta_{\varepsilon}$, then there is an element $w \in M$ such that $\left(M+p^{-1} \mathbf{Z}[w]\right)_{p}$ contains a hyperbolic unimodular plane with $s\left(M+p^{-1} \mathbf{Z}[w]\right) \mathbf{Z}_{p}=\mathbf{Z}_{p}$ and $\min \left(M+p^{-1} \mathbf{Z}[w]\right) \gg p^{1 / 3-2 \varepsilon}\left(p^{-1} \min (M)\right) \geq \min (M)^{1 / 3-2 \varepsilon}$

Proof. Put $S_{1}:=\left\{\left[p^{1 / 3}\right],\left[p^{1 / 3}\right] \pm 1\right\}$ and $S_{2}:=\left\{x \in \mathbf{Z} \mid p^{2 / 3-\varepsilon}<x<p^{2 / 3+\varepsilon}\right\}$. If $p(>5)$ is sufficiently large, then we have $\left|S_{2}\right|>5 \sqrt{p} \log p$, which is supposed in the rest of the proof. By applying Theorem 2 in Section 2 to a quadratic space $V:=M^{\left(p^{-1}\right)} / p M^{\left(p^{-1}\right)}$ over $\mathbf{Z} / p \mathbf{Z}$, there exist a subset $\left\{t_{1}, t_{2}, t_{3}\right\} \subset\{1, \ldots, m\}$, $x_{1}(\equiv \pm 1 \bmod p), x_{2} \bmod p \in S_{1}, x_{3} \bmod p \in S_{2}$ and $y_{i} \in \mathbf{Z}$ for $i \neq t_{j}$, such that $w:=\sum_{j=1}^{3} x_{j} v_{t_{j}}+\sum_{i \neq t_{j}} y_{i} v_{i}$ satisfies $Q(w) \equiv 0 \bmod p^{2}$ and $B(w, M) \not \equiv 0 \bmod p^{2}$. This implies $s\left(M+p^{-1} \mathbf{Z}[w]\right) \mathbf{Z}_{p}=\mathbf{Z}_{p}$, and for an element $u \in M$ with $B(w, u)$ $\not \equiv 0 \bmod p^{2}, \mathbf{Z}_{p}\left[u, p^{-1} w\right]$ is a unimodular hyperbolic plane. Putting $w=\sum r_{i} v_{i}$, we have

$$
\begin{aligned}
& \min \left(M+p^{-1} \mathbf{Z}[w]\right) \\
\asymp & \min \left(\min (M), \min _{p \nmid b} \sum_{i=1}^{m}\left\lceil b r_{i} / p\right\rceil^{2} Q\left(v_{i}\right)\right) \\
\gg & \min \left(\min (M), \min _{p \nmid b} \sum_{j=1}^{3}\left\lceil b x_{j} / p\right\rceil^{2} Q\left(v_{i_{j}}\right)\right) \\
\gg & \min (M) \min \left(1, \min _{p \nmid b} \sum_{j=1}^{3}\left\lceil b x_{j} / p\right\rceil^{2}\right) \\
\gg & \min (M) \min \left(1, \min \left(\left(4 x_{2}^{2}\right)^{-1}, 4^{-1}\left(x_{2} / x_{3}\right)^{2}, p^{-2}\left(1+x_{2}^{2}+x_{3}^{2}\right)\right)\right) \text { by Theorem in }
\end{aligned}
$$


Section 1

$\gg \min (M) \min \left(1, p^{-2 / 3-2 \varepsilon}\right)$

$\gg p^{-2 / 3-2 \varepsilon} \min (M)$.

By putting $\min (M)=p a$, we have $a \geq 1$ and $p^{-2 / 3-2 \varepsilon} \min (M)=\min (M)^{1 / 3-2 \varepsilon}$. $a^{2 / 3+2 \varepsilon} \geq \min (M)^{1 / 3-2 \varepsilon}$.

Lemma 2. Suppose $p \neq 2$. Let $M$ be a positive lattice such that $s(M) \subset p \mathbf{Z}$ and $M_{p}$ is a $p \mathbf{Z}_{p}$-maximal quaternary lattice of ind $\left(\mathbf{Q}_{p} M\right) \leq 1$. Moreover we assume that the rank of a $p$-modular component of $M_{p}$ is at most 2 . Then there is an element $w \in$ $M$ such that $s\left(M+p^{-1} \mathbf{Z}[w]\right) \mathbf{Z}_{p}=\mathbf{Z}_{p}$ and $\min \left(M+p^{-1} \mathbf{Z}[w]\right) \gg p^{1 / 4}$.

Proof. For some integers $\varepsilon_{1}, \varepsilon_{2}$ relatively prime to $p$, we can take a basis $\left\{w_{1}, \ldots, w_{4}\right\}$ of $M$ such that

$$
\left(B\left(w_{i}, w_{j}\right)\right) \equiv \operatorname{diag}\left(p, \varepsilon_{1} p, p^{2}, \varepsilon_{2} p^{2}\right) \bmod p^{3} .
$$

The assumption on $M_{p}$ implies that $-\varepsilon_{2}$ is not a quadratic residue $\bmod p$. For any integers $f, g, s\left(M+p^{-1} \mathbf{Z}\left[f w_{3}+g w_{4}\right]\right) \mathbf{Z}_{p}=\mathbf{Z}_{p}$ is clear, unless $f \equiv g \equiv 0$ $\bmod p$. By putting $s_{i}:=a_{i 3}, t_{i}:=a_{i 4}$ for $a_{i j}$ defined by (1) and $r_{i}:=f s_{i}+g t_{i}$ we have $f w_{3}+g w_{4}=\sum r_{i} v_{i}$ and

$$
\left.\min \left(M+p^{-1} \mathbf{Z}\left[f w_{3}+g w_{4}\right]\right) \asymp \min \left(\min (M), K_{f, g}\right)\right),
$$

where

$$
K_{f, g}:=\min _{b \neq 0 \bmod p} \sum_{i=1}^{4}\left\lceil b r_{i} / p\right\rceil^{2} Q\left(v_{i}\right)
$$

Now we choose $1 \leq \alpha, \beta \leq 4$ by the condition $d_{\alpha, \beta}:=s_{\alpha} t_{\beta}-s_{\beta} t_{\alpha} \not \equiv 0 \bmod p$. Then we have

$$
K_{f, g} \geq \min _{b \neq 0 \bmod p}\left(\left\lceil b r_{\alpha} / p\right\rceil^{2} Q\left(v_{\alpha}\right)+\left\lceil b r_{\beta} / p\right\rceil^{2} Q\left(v_{\beta}\right)\right)
$$

and Corollary 3 in Section 1 with $x_{1}=x_{2}=1, x=0$ there implies the existence of integers $f, g$ such that

$$
K_{f, g} \gg\left(Q\left(v_{\alpha}\right) Q\left(v_{\beta}\right)\right)^{1 / 2} p^{-1}
$$

since $f \equiv g \equiv 0 \bmod p$ is equivalent to $r_{\alpha} \equiv r_{\beta} \equiv 0 \bmod p$. First, suppose $\alpha$ or $\beta \geq 3$; then we have

$$
Q\left(v_{1}\right)^{2} Q\left(v_{2}\right) Q\left(v_{3}\right)=\left(Q\left(v_{1}\right) Q\left(v_{2}\right)\right)\left(Q\left(v_{1}\right) Q\left(v_{3}\right)\right) \ll\left(Q\left(v_{\alpha}\right) Q\left(v_{\beta}\right)\right)^{2} \ll\left(p K_{f, g}\right)^{4} .
$$


On the other hand, we have

$$
Q\left(v_{1}\right)^{2} Q\left(v_{2}\right) Q\left(v_{3}\right) \asymp Q\left(v_{1}\right) d \mathbf{Z}\left[v_{1}, v_{2}, v_{3}\right] \geq p \cdot p^{4}=p^{5},
$$

since elementary divisors of $\left(B\left(w_{i}, w_{j}\right)\right)$ over $\mathbf{Z}_{p}$ are $p, p, p^{2}, p^{2}$. Thus we have $K_{f, g} \gg p^{1 / 4}$ and then $\min \left(M+p^{-1} \mathbf{Z}\left[f w_{3}+g w_{4}\right]\right) \gg p^{1 / 4}$ under the assumption $\alpha$ or $\beta \geq 3$. Next, we suppose that $\alpha$ or $\beta \geq 3$ is impossible; then we have $\{\alpha, \beta\}=$ $\{1,2)$. By the way of choice of $\alpha, \beta$, we have $d_{31} \equiv d_{32} \equiv d_{41} \equiv d_{42} \equiv 0 \bmod p$ and then $s_{3} \equiv t_{3} \equiv s_{4} \equiv t_{4} \equiv 0 \bmod p$. Now we can apply Theorem in Section 3 with $r=2, m=4, t_{1}=t_{2}=1, t_{3}=t_{4}=2, e=1, S=\left(\begin{array}{cc}S_{1}^{(2)} & S_{2} \\ S_{3} & S_{4}\end{array}\right):=\left(B\left(v_{i}, v_{j}\right)\right)$, $D_{1}=\operatorname{diag}\left(p, \varepsilon_{1} p\right), D_{2}=\operatorname{diag}\left(p^{2}, \varepsilon_{2} p^{2}\right)$ and then we have $S_{1}-S_{4}^{-1}\left[S_{3}\right] \equiv S_{3} \equiv 0$ $\bmod p^{2}$ and $S_{4}$ is $p$-modular. Therefore $S_{1} \equiv 0 \bmod p^{2}$ holds and it implies $Q\left(v_{1}\right) \equiv Q\left(v_{2}\right) \equiv 0 \bmod p^{2}$, and by (2) there are integers $f, g$ such that

$$
K_{f, g} \geq p \geq p^{1 / 4} \text {. }
$$

Proposition 1. Let $M$ be a positive lattice such that $\operatorname{rank}(M) \geq 4, s(M) \subset$ $p \mathbf{Z}$. Then there is a positive number $\delta$ satisfying the following condition:

If $p>\delta$, then there is a lattice $M^{\prime}$ containing $M$ such that $\left[M^{\prime}: M\right]$ is a power of prime $p, s\left(M_{p}^{\prime}\right)=\mathbf{Z}_{p}$ and $\min \left(M^{\prime}\right) \geq p^{1 / 4}$. If $\operatorname{rank}(M) \geq 5$ in addition, $M_{p}^{\prime}$ contains a unimodular hyperbolic plane.

Proof. Let a lattice $\bar{M}$ be a lattice such that $[\hat{M}: M]$ is a power of $p$ and $\hat{M}_{p}$ is $p \mathbf{Z}_{p}$-maximal. $\min (\hat{M}) \geq p$ is clear. If $\hat{M}_{p}$ contains a $p$-modular sublattice of rank $\geq 3$, then the assertion follows from Lemma 1 with $\varepsilon=1 / 24$ if $p>\delta_{1 / 24}$. Otherwise, both ind $\left(\mathbf{Q}_{p} M\right) \leq 1$ and $\operatorname{rank}\left(M_{p}\right)=4$ hold and then Lemma 2 implies the assertion.

By virtue of Proposition 1, if $\operatorname{rank}(M) \geq 4$ and $s(M) \subset p \mathbf{Z}$ for a sufficiently large prime number $p$, then there exists a lattice $M^{\prime}(\supset M)$ such that $s\left(M^{\prime}\right) \subset \mathbf{Z}$ and $\min \left(M^{\prime}\right)$ is larger than a given number $\kappa$ in advance. The assumption $m \geq 4$ is crucial. In the following examples, $\min (M)$ is arbitrarily large but $\min \left(M^{\prime}\right) \leq 4+5 p$ for every $M^{\prime}(\supset M)$ with $s\left(M^{\prime}\right) \mathbf{Z}_{p}=\mathbf{Z}_{p}$.

Example 1. Let $M=\mathbf{Z}\left[v_{1}, v_{2}\right]$ be a positive lattice defined by the reduced matrix

$$
\left(B\left(v_{i}, v_{j}\right)\right)=\left(\begin{array}{cc}
p\left(1+p^{s}\right)^{2} & p\left(1+p^{s}\right) \\
p\left(1+p^{s}\right) & p+4(1+p) p^{2 s}
\end{array}\right)
$$


where $p$ is an odd prime number and $s$ is a natural number. Then $d(M)=4(1+p)$ $\left(1+p^{s}\right)^{2} p^{2 s+1}$ and $M_{p} \cong\langle p\rangle \perp\left\langle p^{2 s}\right\rangle$. Moreover, by putting $M^{\prime}:=M+\mathbf{Z}\left[p^{-t} w\right]$ for $w \in M$, the condition $s\left(M_{p}^{\prime}\right)=\mathbf{Z}_{p}$ compels $M^{\prime}=\mathbf{Z}\left[p^{-s}\left(v_{1}-v_{2}\right), v_{1}\right]$ and then $\min \left(M^{\prime}\right) \leq Q\left(p^{-s}\left(v_{1}-v_{2}\right)\right)=4+5 p$.

Example 2. Let $M=\mathbf{Z}\left[v_{1}, v_{2}\right] \perp \mathbf{Z}\left[v_{3}\right]$, where $v_{1}, v_{2}$ are those in Example 1 and $Q\left(v_{3}\right):=a p$, where $a$ is a natural number relatively prime to $p$ satisfying that $a>\left(1+p^{s}\right)^{2}$ and $-a$ is not a square in $\mathbf{Z}_{p}$. Then we have $\min (M)=p(1+$ $\left.p^{s}\right)^{2}$ and by putting $M^{\prime}:=M+\mathbf{Z}\left[p^{-t} w\right]$ for $w \in M$, the condition $s\left(M_{p}^{\prime}\right)=\mathbf{Z}_{p}$ compels $M^{\prime}=\mathbf{Z}\left[p^{-s}\left(v_{1}-v_{2}\right), v_{1}, v_{3}\right]$ and then $\min \left(M^{\prime}\right) \leq Q\left(p^{-s}\left(v_{1}-v_{2}\right)\right)=4$ $+5 p$

Example 3. Let $v_{1}, v_{2}$ and $v_{3}$ be as in the previous example. Put $M:=\mathbf{Z}\left[v_{1}\right.$, $\left.v_{2}\right] \perp \mathbf{Z}\left[v_{3}\right] \perp\left(\perp_{i=4}^{m-3} \mathbf{Z}\left[v_{i}\right]\right)$ with $Q\left(v_{\imath}\right)>a\left(1+p^{s}\right)^{2}$ and put $Q\left(v_{i}\right) \in\left(\mathbf{Z}_{p}^{\times}\right)^{2}$ for $i \geq 4$; then if, for a lattice $\hat{M} \supset M, \hat{M}_{p}$ is primitively represented by $N_{p}=$ $\left\langle 1_{m}\right\rangle \perp\langle-1\rangle \perp\langle-1\rangle \perp\langle-\delta\rangle\left(\delta \in \mathbf{Z}_{p}^{\times} \backslash\left(Z_{p}^{\times}\right)^{2}\right)$, then we have $\hat{M}=\mathbf{Z}\left[p^{-s}\left(v_{1}\right.\right.$ $\left.\left.-v_{2}\right), v_{1}, v_{3}, \ldots, v_{m}\right]$ and $\min (\bar{M}) \leq 4+5 p$.

In Example 3, a local extension of $M$ is uniquely determined under the condition that it is primitively represented by $N_{p}$. If this is not the case, is there an extension $M^{\prime}$ with $\min (M)$ being small? If so, we can make a counter-example to the assertion $A_{m, n}$.

Lemma 3. Let $p$ be an odd prime and $F_{p}:=\mathbf{Z} / p \mathbf{Z}$. Suppose that $V$ be a quadratic space over $F_{p}$ with basis $\left\{z_{1}, \ldots, z_{t}\right\}$ and integers $r_{1}=1, r_{2}, \ldots, r_{t}$ are given. If $Q(V) \neq\{0\}$, then there are integers $x_{1}=r_{1}(=1), x_{i}=r_{i}, r_{\imath} \pm 1(i>1)$ satisfying $Q\left(\sum_{i=1}^{t} x_{i} z_{i}\right) \neq 0$.

Proof. We use induction on $t$. The case of $t=1$ is clear. Suppose that the assertion is false for $t>1$. Since the equation

$$
Q\left(\sum_{i=1}^{t} x_{i} z_{i}\right)=x_{t}^{2} Q\left(z_{t}\right)+2 x_{t}\left(\sum_{i=1}^{t-1} B\left(z_{t}, z_{i}\right) x_{\imath}\right)+Q\left(\sum_{i=1}^{t-1} x_{i} z_{i}\right)=0,
$$

has the three solutions $x_{t}=r_{t}, r_{t} \pm 1$, we have

$$
Q\left(z_{t}\right)=0, \sum_{i=1}^{t-1} B\left(z_{t}, z_{\imath}\right) x_{i}=0, Q\left(\sum_{i=1}^{t-1} x_{i} z_{i}\right)=0,
$$

for $x_{1}=1, x_{i}=r_{i}, r_{i} \pm 1$ for $i=2, \ldots, t-1$. From the induction hypothesis, 
$Q\left(F_{p}\left[z_{1}, \ldots, z_{t-1}\right]\right)=0$ follows. Making use of the middle equality above for $x_{\imath}=$ $r_{i}, r_{i}+1$, we have $B\left(z_{t}, z_{i}\right)=0$ for $i=2, \ldots, t-1$ and hence $B\left(z_{t}, z_{1}\right)=0$. Thus we have the contradiction $Q(V)=\{0\}$.

Lemma 4. Let $L=\mathbf{Z}_{p}\left[w_{1}, \ldots, w_{t}\right]$ be a quadratic lattice over $\mathbf{Z}_{p}$ such that $\left(B\left(w_{i}, w_{j}\right)\right)=\operatorname{diag}\left(\varepsilon_{1} p^{a_{1}}, \ldots, \varepsilon_{t} p^{a_{t}}\right),\left(\varepsilon_{i} \in \mathbf{Z}_{p}^{\times}, a_{1}=0 \leq a_{2} \leq \cdots \leq a_{t}\right)$, and assume $a_{1}<a_{2}$ if $p=2$. Let $\left\{z_{1}, \ldots, z_{t}\right\}$ be another basis of $L$ and let $r_{1}=1, r_{2}, \ldots$, $r_{t}$ be integers. Then for integers $x_{1}=1, x_{i}=r_{i}, r_{i} \pm 1(i>1)$, we have $Q\left(\sum_{i=1}^{t}\right.$ $\left.x_{i} z_{i}\right) \in \mathbf{Z}_{p}^{\times}$.

Proof. If $p \neq 2$, then we have only to apply Lemma 3 to $L / p L$. Suppose $p=2$ and ${ }^{t}\left(z_{1}, \ldots, z_{t}\right)=B^{t}\left(w_{1}, \ldots, w_{t}\right)$ for some $B \in G L_{t}\left(\mathbf{Z}_{2}\right)$. By virtue of $\sum_{\imath=1}^{t} x_{i} z_{\imath}=\left(x_{1}, \ldots, x_{t}\right) B^{t}\left(w_{1}, \ldots, w_{t}\right)$, we have only to show that $\sum_{t=1}^{t} x_{i} b_{i 1} \not \equiv$ $0 \bmod 2$, which implies $Q\left(\sum_{i=1}^{t} x_{i} z_{i}\right) \in \mathbf{Z}_{2}^{\times}$. If $\sum_{i=1}^{t} x_{i} b_{i 1} \equiv 0 \bmod 2$ for $x_{1}=1$, $x_{i}=r_{i}, r_{i}+1(i>1)$, we have $b_{i 1} \equiv 0 \bmod 2$ for $i \geq 1$, which is the contradiction.

Lemma 5. Let $p$ be a prime number and $M$ a positive lattice of $\operatorname{rank}(M)=m$, $s(M) \subset \mathbf{Z}$. Suppose that

$$
M_{p} \cong\left\langle\operatorname{diag}\left(\varepsilon_{1} p^{a_{1}}, \ldots, \varepsilon_{m} p^{a_{m}}\right)\right\rangle
$$

where $\varepsilon_{i} \in \mathbf{Z}_{p}^{\times}$and $0 \leq a_{1} \leq \cdots \leq a_{m}$. Divide the set $\{1, \ldots, m\}$ to disjoint subsets $S$ and $T:=\left\{h_{1}, \ldots, h_{m-r}\right\}\left(h_{1}<\cdots<h_{m-r}\right.$ and $\left.0 \leq r:=|S|<m\right)$, and suppose $a_{h_{i}}<a_{h_{2}}$ if $p=2$ and let $s$ be a natural number $\leq a_{h_{1}} / 2$. Let $\left\{w_{1}, \ldots, w_{m}\right\}$ be a basis of $M$ such that $\left(B\left(w_{i}, w_{j}\right)\right)$ is sufficiently close to $\operatorname{diag}\left(\varepsilon_{1} p^{a_{1}}, \ldots, \varepsilon_{m} p^{a_{m}}\right)$ in $M_{m}\left(\mathbf{Z}_{p}\right)$. Let $A=\left(a_{i j}\right)$ be the transformation matrix defined by (1), and choose integers $k_{1}<\cdots<k_{m-r}$ so that the determinant of $\left(a_{k_{i}, h_{j}}\right)_{1 \leq i, j \leq m-r}$ is relatively prime to p. Then there are integers $f_{i}(i \in T)$ such that for $w=\sum_{i \in T} f_{i} w_{i}$ we have

$$
\begin{gathered}
\min \left(M+p^{-s} \mathbf{Z}[w]\right) \gg\left(p^{-2 s} \prod_{i=1}^{m-r} Q\left(v_{k_{i}}\right)\right)^{1 /(m-r)}, \\
\left(M+p^{-s} \mathbf{Z}[w]\right)_{p} \cong\left(\perp_{i \in S}\left\langle\varepsilon_{i} p^{a_{i}}\right\rangle\right) \perp\left\langle\varepsilon_{h_{1}} p^{a_{h_{1}}-2 s}\right\rangle \perp K_{p},
\end{gathered}
$$

for some lattice $K_{p}$ of $\operatorname{rank}\left(K_{p}\right)=m-r-1$ and $s\left(K_{p}\right) \subset p^{a_{h_{2}}} \mathbf{Z}_{p}$. If $r \leq m / 2-1$ in addition, then we have $\min \left(M+\mathbf{Z}\left[p^{-s} w\right]\right) \gg \min (M)^{1 /(m-r)}$.

Proof. Let $r_{1}, \ldots, r_{m-r}$ be integers, and for $B=\left(a_{k_{i} h_{j}}\right)_{1 \leq i, j \leq m-r}$ we define integers $f_{h_{i}}$ by 


$$
\left(f_{h_{1}}, \ldots, f_{h_{m-r}}\right) \equiv\left(r_{1}, \ldots, r_{m-r}\right)^{t} B^{-1} \bmod p^{s} .
$$

By putting $R_{i}:=\sum_{j \in T} a_{i j} f_{j}$, we have

$$
\begin{gathered}
{ }^{t}\left(R_{k_{1}}, \ldots, R_{k_{m-r}}\right)=B^{t}\left(f_{h_{1}}, \ldots, f_{h_{m-r}}\right) \equiv{ }^{t}\left(r_{1}, \ldots, r_{m-r}\right) \bmod p^{s} \\
\min \left(M+\mathbf{Z}\left[p^{-s} w\right]\right) \asymp \min \left(\min (M), \min _{p^{s} \ngtr b} \sum_{i=1}^{m}\left\lceil b R_{i} / p^{s}\right\rceil^{2} Q\left(v_{i}\right)\right) \\
\min _{p^{s} \nmid b} \sum_{i=1}^{m}\left\lceil b R_{i} / p^{s}\right\rceil^{2} Q\left(v_{i}\right) \gg \min _{p^{s} \Varangle b} \sum_{i=1}^{m-r}\left\lceil b r_{i} / p^{s}\right\rceil^{2} Q\left(v_{k_{i}}\right) .
\end{gathered}
$$

Since $Q\left(v_{k_{1}}\right) \ll \cdots \ll Q\left(v_{k_{m-r}}\right)$, Corollary 1 in Section 1 yields that there exist integers $r_{m-r}=1, r_{m-r-1}, \ldots, r_{1}$ such that

$$
\min _{p^{s} \ngtr b} \sum_{i=1}^{m-r}\left\lceil b r_{i} / p^{s}\right\rceil^{2} Q\left(v_{k_{i}}\right) \gg\left(p^{-2 s} \prod_{i=1}^{m-r} Q\left(v_{k_{i}}\right)\right)^{1 /(m-r)} \text {. }
$$

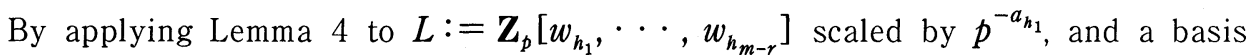
${ }^{t}\left(z_{1}, \ldots, z_{m-r}\right):={ }^{t} B^{-1 t}\left(w_{h_{1}}, \ldots, w_{h_{m-r}}\right)$, there exist integers $r_{m-r}^{\prime}=1, r_{i}^{\prime}=r_{i}$ or $r_{i} \pm 1(1 \leq i<m-r)$ such that $\operatorname{ord}_{p} Q\left(\sum_{i} r_{i}^{\prime} z_{i}\right)=a_{h_{1}}$. Define integer $f_{h_{i}}^{\prime}$ by $\left(f_{h_{1}}^{\prime}, \cdots, f_{h_{m-r}}^{\prime}\right) \equiv\left(r_{1}^{\prime}, \cdots, r_{m-t}^{\prime}\right)^{t} B^{-1} \bmod p^{s}$; then $w^{\prime}:=\sum_{i} f_{h_{i}}^{\prime} w_{h_{i}} \equiv \sum_{i} r_{i}^{\prime} z_{i} \bmod$ $p^{s} L$ and hence $\operatorname{ord}_{p} Q\left(w^{\prime}\right)=a_{h_{1}}$. Thus we may assume $\operatorname{ord}_{p} Q(w)=a_{h_{1}}$. Hence $w$ splits $\mathbf{Z}_{p}\left[w_{i}(i \in T)\right]$, and

$$
K_{p}=\mathbf{Z}_{p}\left[w_{i}-\frac{B\left(w, w_{i}\right)}{Q(w)} w \mid i \in T\right]
$$

which implies the second assertion. Finally we assume $m \geq 2 r+2$; then we have $\Pi_{i=1}^{m-r-1} Q\left(v_{k_{i}}\right) \asymp \mathrm{d} \mathbf{Z}\left[v_{k_{1}}, \ldots, v_{k_{m-r-1}}\right] \geq p^{a_{1}+\cdots+a_{m-r-1}} \geq p^{a_{m-r-1}} \geq p^{a_{r+1}} \geq p^{a_{h_{1}}}$ since $m \geq h_{m-r}>\cdots>h_{1}$ implies $m-r \geq h_{m-r}-r>\cdots>h_{1}-r$ and hence $h_{1}$ $-r \leq 1$.

Remark. In Lemma 5, the assumption $a_{h_{1}}<a_{h_{2}}$ is not satisfied in general. But we can modify it by enlarging as follows: If $a_{h_{1}}=a_{h_{2}}$, then for $M^{\prime}:=M+$ $\mathbf{Z}\left[w_{h_{1}} / p\right]$ we have $\min \left(M^{\prime}\right) \asymp_{p} \min (M)$ and $M_{p}^{\prime} \cong\left\langle\operatorname{diag}\left(\varepsilon_{1} p^{a_{1}}, \ldots, \varepsilon_{h_{1}-1} p^{a_{h_{1}-1}}\right.\right.$, $\left.\left.\varepsilon_{h_{1}} p^{a_{h_{1}}-2}, \varepsilon_{h_{1}+1} p^{a_{h_{1}+1}}, \ldots, \varepsilon_{m} p^{a_{m}}\right)\right\rangle \perp K_{p}$.

When $p=2$, a lattice does not have any orthogonal basis in general, but the following is useful to reduce to a lattice having an orthogonal basis. If $H_{2}=\mathbf{Z}_{2}\left[w_{1}\right.$, $\left.w_{2}\right]$ is isometric to $\left(B\left(w_{i}, w_{j}\right)\right)=2^{a}\left(\begin{array}{ll}0 & 1 \\ 1 & 0\end{array}\right)$, then

$$
H_{2}+\mathbf{Z}_{2}\left[\left(w_{1}+w_{2}\right) / 2\right]=\mathbf{Z}_{2}\left[\left(w_{1}+w_{2}\right) / 2,\left(w_{1}-w_{2}\right) / 2\right] \cong\left\langle\operatorname{diag}\left(2^{a-1},-2^{a-1}\right)\right\rangle .
$$


If $\left(B\left(w_{i}, w_{j}\right)\right)=2^{a}\left(\begin{array}{ll}2 & 1 \\ 1 & 2\end{array}\right)$, then

$$
H_{2}+\mathbf{Z}_{2}\left[w_{1} / 2\right]=\mathbf{Z}_{2}\left[w_{1} / 2, w_{2}-w_{1} / 2\right] \cong\left\langle\operatorname{diag}\left(2^{a-1}, 3 \cdot 2^{a-1}\right)\right\rangle \text {. }
$$

Lemma 6. Let $0 \leq r \leq m \leq n$ be integers and $M=K_{1} \perp K_{2}$, $N$ be regular quadratic lattices over $\mathbf{Z}_{p}$ with $\operatorname{rank}(M)=m, \operatorname{rank}\left(K_{1}\right)=r$ and $\operatorname{rank}(N)=n$. (If $r$ $=0$, then we assume $\left.K_{1}=0\right)$ Suppose that there is a quadratic space $V$ such that $\mathbf{Q}_{p} N \cong \mathbf{Q}_{p} K_{1} \perp V$ and $\operatorname{ind}(V) \geq m-r$, and that $M$ is represented by $N$. Then there is a constant $c=c\left(K_{1}, N\right)$ such that there is a lattice $M^{\prime}$ in $N$ isometric to $M$ with $\left[\mathbf{Q}_{p} M^{\prime} \cap N: M^{\prime}\right]<c$.

Proof. Put $S:=\left\{K \subset N \mid K \cong K_{1}\right\}$ and let $\left\{H_{1}, \ldots, H_{t}\right\}$ be the set of representatives of $O(N) \backslash S$. Since $M$ is represented by $N$, there exist an isometry $\sigma$ from $M$ to $N$ and an integer $i(1 \leq i \leq t)$ so that $\sigma\left(K_{1}\right)=H_{i}$. By virtue of $\mathbf{Q}_{p} H_{i}$ $\cong \mathbf{Q}_{p} K_{1}$, we have $\mathbf{Q}_{p} H_{i}^{\perp} \cong V$ and hence ind $\left(\mathbf{Q}_{p} H_{i}^{\perp}\right) \geq m-r$. Since $K_{2}$ is represented by $H_{i}^{\perp}$, we can apply Lemma 3 in [2] and therefore there is a constant $c_{i}$ such that there is a lattice $K_{2}^{\prime}\left(\subset H_{i}^{\perp}\right)$ satisfying $K_{2}^{\prime} \cong K_{2}$ and $\left[\mathbf{Q}_{p} K_{2}^{\prime} \cap H_{i}^{\perp}\right.$ : $\left.K_{2}^{\prime}\right]<c_{\imath}$. Now $M^{\prime}:=H_{i} \perp K_{2}^{\prime}(\cong M)$ satisfies

$$
\begin{aligned}
{\left[\mathbf{Q}_{p} M^{\prime} \cap N: M^{\prime}\right] } & =\left[\mathbf{Q}_{p} M^{\prime} \cap N: \mathbf{Q}_{p} M^{\prime} \cap\left(H_{i} \perp H_{i}^{\perp}\right)\right]\left[\mathbf{Q}_{p} M^{\prime} \cap\left(H_{i} \perp H_{i}^{\perp}\right): M^{\prime}\right] \\
& \leq\left[N: H_{i} \perp H_{i}^{\perp}\right]\left[H_{i} \perp\left(\mathbf{Q}_{p} K_{2}^{\prime} \cap H_{i}^{\perp}\right): H_{\imath} \perp K_{2}^{\prime}\right] \\
& \leq\left[N: H_{i} \perp H_{i}^{\perp}\right] c_{\imath} .
\end{aligned}
$$

Thus the number $c\left(K_{1}, N\right):=\max _{i}\left[N: H_{i} \perp H_{i}^{\perp}\right] c_{i}$ is what we want.

Proposition 2. Let $M$ and $N$ be positive lattices of $\operatorname{rank}(M)=m$ and $\operatorname{rank}(N)=n$ respectively, and $p$ a prime number, and suppose that $n \geq 2 m-[m / 2]$ +3 and $M_{p}$ is represented by $N_{p}$. Then there is a lattice $M^{\prime}(\supset M)$ such that $M_{q}^{\prime}=$ $M_{q}$ if $q \neq p, M_{p}^{\prime}$ is primitively represented by $N_{p}$ and $\min \left(M^{\prime}\right)>c\left(N_{p}\right) \min (M)^{c_{p}}$, where $c\left(N_{p}\right)$ depends only on $N_{p}$ and $c_{p}$ depends only on $m$.

Proof. First, we note that if once, for a lattice $\hat{M} \supset M$, an isometry $\sigma$ from $\bar{M}_{p}$ to $N_{p}$ with $\left[\mathbf{Q}_{p} \sigma\left(\hat{M}_{p}\right) \cap N_{p}: \sigma\left(\hat{M}_{p}\right)\right]<c$ has been constructed, then $\hat{M}$ has an extension $M^{\prime}$ such that $M_{p}^{\prime}=\sigma^{-1}\left(\mathbf{Q}_{p} \sigma\left(\hat{M}_{p}\right) \cap N_{p}\right)$ is primitively represented by $N_{p}, M_{q}^{\prime}=\bar{M}_{q}$ for $q \neq p$ and $\left[M^{\prime}: \hat{M}\right]<c$, which yields $\min \left(M^{\prime}\right)>c^{-2} \min (\hat{M})$. Let $h_{1}$ be an integer such that $N_{p}$ contains a $p^{h_{1}} \mathbf{Z}_{p}$-maximal lattice.

Let $h$ be an integer and $S(h)$ the set of regular submodules $K_{p}$ of $N_{p}$ such that the scale of each Jordan component of $K_{p}$ contains $p^{h} \mathbf{Z}_{p}$. Then there is a finite subset 
$X(h)$ of $S(h)$ so that any $L \in S(h)$ is transformed to an element in $X(h)$ by $O\left(N_{p}\right)$. Hence we can define an integer $n(h)\left(>h_{1}\right)$ so that for $L \in S(h), L^{\perp}$ in $N_{p}$ contains a maximal lattice whose norm contains $p^{n(h)} \mathbf{Z}_{p}$. We note that $n(h)$ depends only on $h$ and $N_{p}$.

First, suppose $s\left(M_{p}\right) \subset p^{h_{1}+2} \mathbf{Z}_{p}$; then by the iterative application of Lemma 5 and the remark after it for $p=2$, there is a lattice $\hat{M}(\supset M)$ such that $\min (\hat{M}) \gg_{p}$ $\min (M)^{c_{p}}$ and $\bar{M}_{p} \cong\left\langle\operatorname{diag}\left(\varepsilon_{1} p^{a_{1}}, \ldots, \varepsilon_{m} p^{a_{m}}\right)\right\rangle$ with $h_{1} \leq a_{1} \leq \cdots \leq a_{m}$ and $a_{[m / 2]}$ $\leq h_{1}+1$. Since $N_{p}$ contains $p^{h_{1}} \mathbf{Z}_{p}$-maximal lattice, $\hat{M}_{p}$ is represented by $N_{p}$. We note that for a regular quadratic space $V$ over $\mathbf{Q}_{p}, \operatorname{dim}(V) \geq 2 t+3$ implies $\operatorname{ind}(V) \geq t$. By applying Lemma 6 to $\hat{M}_{p}=K_{1} \perp K_{2}$ where $K_{1} \cong\left\langle\operatorname{diag}\left(\varepsilon_{1} p^{a_{1}}, \ldots\right.\right.$, $\left.\left.\varepsilon_{[m / 2]} p^{a_{[m / 2]}}\right)\right\rangle$ and $K_{2} \cong\left\langle\operatorname{diag}\left(\varepsilon_{[m / 2]+1} p^{a_{[m / 2]+1}}, \ldots, \varepsilon_{m} p^{a_{m}}\right)\right\rangle$, there is a constant $c\left(h_{1}\right.$, $\left.N_{p}\right)$ such that there is an isometry $\sigma$ from $\bar{M}_{p}$ to $N_{p}$ such that $\left[\mathbf{Q}_{p} \sigma\left(\hat{M}_{p}\right) \cap N_{p}\right.$ : $\left.\sigma\left(\hat{M}_{p}\right)\right]<c\left(h_{1}, N_{p}\right)$.

Next suppose that $M_{p}=J_{1} \perp J_{2}$ with $\operatorname{rank}\left(J_{1}\right)=r$ and that the scale of every Jordan component of $J_{1}$ contains $p^{h} \mathbf{Z}_{p}$ and $s\left(J_{2}\right) \subset p^{h+1} \mathbf{Z}_{p}$ with an integer $h$ $\leq h_{1}+1$. If $s\left(J_{2}\right) \subset p^{n(h)} \mathbf{Z}_{p}$ and $r \leq[m / 2]-1$, then by virtue of Lemma 5 , there exists a lattice $\hat{M}$ ( $\supset M$ ) such that $\min (\hat{M}) \gg \min (M)^{1 /(m-r)}$, and $\hat{M}_{p} \cong J_{1}$ $\perp\left\langle\varepsilon p^{n(h)+\delta}\right\rangle \perp K_{p}$ for $\varepsilon \in \mathbf{Z}_{p}^{\times}, \delta=0$ or 1 and some lattice $K_{p}$ of $s\left(K_{p}\right) \subset p^{n(h)} \mathbf{Z}_{p}$. By virtue of the choice of $n(h), \bar{M}_{p}$ is represented by $N_{p}$. Thus by iterating this, there exists a lattice $\bar{M} \supset M$ such that (i) $\min (\bar{M}) \gg \min (M)^{c}$ for some positive number $c$ dependent only on $m$, (ii) $\bar{M}_{p}=\left\langle\varepsilon_{1} p^{a_{1}}\right\rangle \perp \cdots \perp\left\langle\varepsilon_{m} p^{a_{m}}\right\rangle$ with $a_{1} \leq \cdots$ $\leq a_{m}$ and $a_{i+1}-a_{i}<c_{p}(N)$ for some positive number dependent on $N_{p}$ for $i \leq[m / 2]-1$, and (iii) $\bar{M}_{p}$ is represented by $N_{p}$. Now we can apply Lemma 6 with $r=[m / 2]$ because of $n-[m / 2] \geq 2(m-[m / 2])+3$, and complete the proof.

Proof of Theorem. Let $M$ and $N$ be positive lattices of $\operatorname{rank}(M)=m$ and $\operatorname{rank}(N)=n$ and suppose that $M_{p}$ is represented by $N_{p}$ for every prime $p$. We note that $M_{p}$ is primitively represented by $N_{p}$ if and only if $M_{p} / p M_{p}$ is represented by $N_{p} / p N_{p}$ over $\mathbf{Z}_{p} / p \mathbf{Z}_{p}$ when $N_{p}$ is umimodular and $p>2$. We assume that $s(N) \subset \mathbf{Z}$ without loss of generality. Let $\delta$ be a natural number given in Proposition 1 and we assume that $N_{p}$ is unimodular if $p>\delta$.

(i) Suppose that there is a prime $p$ such that $s\left(M_{p}\right) \subset p \mathbf{Z}_{p}$ and $p>\delta$. By enlarging $M$ to $M^{\prime}$, we may assume that $M_{q}^{\prime}$ is primitively represented by $N_{q}$ if $q \neq p$ and $M_{p}^{\prime}=M_{p}$. If $m \geq 4$, then we can use Proposition 1 and conclude that there is a lattice $\hat{M} \supset M^{\prime}$ such that $s\left(\hat{M}_{p}\right)=\mathbf{Z}_{p}$ and $\min (\hat{M}) \geq p^{1 / 4}$. If $n=2 m$ in addition, the condition $s\left(\hat{M}_{p}\right)=\mathbf{Z}_{p}$ implies that $\hat{M}_{p}$ is primitively represented by $N_{p}$. (If $n<2 m$, then the property $s\left(\bar{M}_{p}\right)=\mathbf{Z}_{p}$ does not yield the primitively- 
representedness of $\hat{M}_{p}$ by $N_{p}$.)

(ii) Denote by $S$ the set of primes $p$ such that $M_{p}$ is not primitively represented by $N_{p}$. Excluding the case (i), we assume that the condition $s\left(M_{p}\right) \subset p \mathbf{Z}_{p}$ yields $p<\delta$ and hence $S \subset\{p \mid p<\delta\}$ by $n=2 m$. If $n \geq 2 m-[m / 2]+3=m+[(m+$ $1) / 2]+3$, then by iterative use of Proposition 2 , there is a lattice $\bar{M}(\supset M)$ such that $\min (\hat{M})>c(N) \min (M)^{c}$ for some constants $c(N), c$ where $c(N)$ depends on $N$ but $c$ does not depend on $M, N$.

Remark. Let us examine the above proof in the case of $\operatorname{rank}(N)=2 m-1$. We assume $m \geq 5$; then at the step (i), we may assume that $\hat{M}_{p}$ contains a unimodular hyperbolic plane and hence $\hat{M}_{p}$ is primitively represented by $N_{p}$. Thus we can clear the case (i). But at the setp (ii), the cardinality of the set $S$ is not less than a constant independent of $M$. So, after applying Proposition 2 iteratively, $\min (\hat{M})$ may be small.

\section{REFERENCES}

[1] L-K. Hua, Introduction to number theory, Springer-Verlag, 1982.

[2] Y. Kitaoka, Local densities of quadratic forms, in "Advanced Studies in Pure Mathematics, 13 (Investigation in Number Theory)" (1988), 433-460.

[3] - Arithmetic of quadratic forms, Cambridge University Press, 1993.

[4] - The minimum and the primitive representation of positive definite quadratic forms, Nagoya Math. J., 133 (1994), 127-153.

[5] W. M. Schmidt, Equations over Finite Fields. An elementary Approach, Springer Lecture Notes in Math., vol. 536, Springer-Verlag. 1976.

[6] I. M. Vinogradov, Elements of Number Theory, Dover Publications, 1954.

Graduate School of Polymathematics

Nagoya University

Chikusa-ku, Nagoya 464-01

Japan 